\title{
A multiple regime nonlinear asymmetric
}

\section{$\operatorname{AR}(\mathrm{p})-\mathrm{GARCH}(1,1)$ model}

\author{
Adán Díaz Hernández \\ EGADE Business School, Instituto Tecnológico y de Estudios \\ Superiores de Monterrey, Tlalpan 14380, Mexico \\ Nick Constantinou* \\ Essex Business School, University of Essex, Colchester CO4 3SQ, UK
}

July 26, 2011

\footnotetext{
* Corresponding author: Nick Constantinou. Tel +44 01206873919.

E-mail addresses: adan.diaz@itesm.mx (A. Díaz), nconst@essex.ac.uk (N. Constantinou).

Adán Díaz Hernández would like to thank the Essex Business School for financial support through its Research Committee Award and its hospitality.
} 


\begin{abstract}
In this article a multiple regime extension of a Heston-Nandi GARCH(1,1) type model is presented to describe the asymmetries and intermittent dynamics in financial volatility. The statistical properties and the estimation of their parameters are addressed in detail. The number of regimes in the model is determined through a statistical procedure based on a robust Lagrange Multiplier (LM) specification. The ability of the model to forecast financial market volatility is empirically compared to other GARCH models for a set comprising some of the major world stock indexes and their corresponding foreign exchange rates during the recent financial crisis.
\end{abstract}

Key words: GARCH, Regime-switching, volatility forecast, risk measurement

JEL Classification: C13, C51, C53

\title{
1 Introduction
}

The problem of modeling and forecasting the volatility of financial time series is one of the major tasks in investment, security valuation, risk management, monetary policy among other areas in economics and quantitative finance. As a result of the seminal papers by Engle (1982) and Bollerslev (1986), the generalized autoregressive conditional heteroskedasticity (GARCH) model has been one of the most popular and widely used solutions for tackling this problem. Despite the ability of GARCH models to capture the so-called volatility clustering and their relatively accurate volatility forecasts (Andersen and Bollerslev 1998), some of their main drawbacks are the symmetry in the response of volatility to past shocks and the sometimes very high implied volatility that are predicted. Regarding the former, as highlighted by Black (1976) and discussed by Christie (1982) and others, it has been observed that negative stock returns are followed by larger increases in volatility than equally large positive returns. This behavior is commonly referred to as the leverage effect, a stylized fact not well captured by the original GARCH models. The leverage effect is related to a rise 
in the debt to equity ratio of the firm leading to increased volatility and hence specific to stocks. The second issue is related to the observed exaggerated volatility persistence implied by these models compared to the true volatility process. This discrepancy has been verified in exchange rates and stock returns (Engle and Mustafa 1992) and seems to be particularly pronounced after extreme shocks such as stock market crashes.

To incorporate asymmetry, different volatility models have been proposed in the literature. The exponential GARCH (EGARCH) model proposed by Nelson (1991) introduces asymmetry in the dynamics of the natural logarithm of the conditional variance according to the sign of past returns. In the GJR model introduced by Glosten, Jagannanthan, and Runkle (1993), the effect of past squared returns on the current conditional variance depends on the sign of the lagged returns. Several alternative specifications are encompassed by the asymmetric power ARCH (APARCH) model proposed by Ding, Granger and Engle (1993).

As an alternative approach, regime-switching models are widely used by both practitioners and researchers to capture changes in regime and nonlinearities in economic and financial variables. In the context of the classical regression model, Goldfeld and Quandt (1973) introduced a version of switching regressions, which have been referred to subsequently as Markov-Switching models. More recently, following the seminal paper by Hamilton (1989), dynamic versions of these models, in which a latent (unobservable) state variable controls the regime shifts follows a Markov-chain, have been widely studied. These models are successful in capturing the time series behavior of many financial variables, resulting in significant research output in this area.

Meanwhile, the smooth transition approach is an alternative method of incorporating changes of regime in time series models through observable variables. Several models have been proposed in the literature. For example, the volatility-switching GARCH (VGARCH) model by Fornari and Mele (1997) is a generalization of the GJR model in which all the coefficients change according to the sign of past returns. More recently, the flexible coefficient GARCH (FCGARCH) model by Medeiros and Veiga (2009) which nests several of the above 
specifications and, based on the smooth transition procedure, is defined by applying the logistic distribution as a weighting factor to multiple $\operatorname{GARCH}(1,1)$ dynamics.

In this paper we propose a new multi-regime non-linear asymmetric GARCH (MRNGARCH) model, within the smooth transition framework, that allows the persistence in conditional volatility to depend on its level as well as incorporating multiple limiting regimes to describe intermittent dynamics of volatility. This model relates very closely to the GARCH(1,1) model by Heston and Nandi (2000) allowing for the inclusion of several limiting regimes. The motivation for extending this $\mathrm{GARCH}$ specification relies on its potential and attractive applicability for pricing derivatives through a model that adequately captures the price dynamics of the underlying asset. The development of an option pricing framework underpinned by the MRNGARCH model is left for future research.

The MNRGARCH model is demonstrated to have the following advantages. First, the stationarity restriction on the model parameters allows for rich dynamics. Both explosive and stable limiting regimes can be included for a better description of the extreme movements and stylized facts of financial time series that standard GARCH specifications fail to capture. The second advantage is that more than two limiting regimes can be modeled and the number of regimes is determined by a sequential hypothesis testing procedure using a Lagrange Multiplier (LM)-statistic. This procedure circumvents the identification problem by using a novel approximation to the so called lower incomplete gamma function and a robust test under non-normality.

The stationarity and ergodicity of the derived process and the problem of parameter estimation are addressed. The consistency and asymptotic normality of the quasi-maximum likelihood estimators (QMLEs) are left for future research, however some remarks on a feasible approach are discussed. In addition, an empirical exercise employing 18 stock indexes and 13 foreign exchange (FX) rates return against U.S. dollar (USD) is implemented to evaluate the ability of the MRNGARCH for modeling and forecasting volatility.

The rest of the paper is organized as follows. Section 2 introduces the model and its 
statistical properties are analyzed. Section 3 discusses in detail the estimation of the MRNGARCH model and the hypothesis testing procedure to determine the number of limiting regimes. In section 4 the model is applied to analyze the return series of a set of stock indices and their related FX rates to USD. Section 5 contains concluding remarks. Technical results and their proofs are given in the Appendix.

\section{The MRNGARCH Model}

To define the model, consider a stochastic process $\left(X_{t}\right)_{t \in \mathbb{Z}}$ defined on some filtered probability space $\left(\Omega, \mathcal{F}, P,\left(\mathcal{F}_{t}\right)_{t \in \mathbb{Z}}\right)$, where the available information up to time $t$ is represented by the sigma algebra $\mathcal{F}_{t}$. Conditional on $\mathcal{F}_{t-1}$, the conditional mean and variance of the process are denoted by $\mu_{t} \equiv E\left(X_{t} \mid \mathcal{F}_{t-1}\right)$ and $\sigma_{t}^{2} \equiv \operatorname{Var}\left(X_{t} \mid \mathcal{F}_{t-1}\right)$, respectively in what follows.

Definition 1. The error process $u_{t} \equiv X_{t}-\mu_{t}$ is said to follow a first-order multipleregime nonlinear asymmetric $G A R C H$ model with $m$ limiting regimes, $M R N G A R C H(m, 1,1)$ if

$$
\begin{aligned}
u_{t}= & \sigma_{t} \varepsilon_{t}, \\
\sigma_{t}^{2}= & \alpha_{0}+\beta_{0} \sigma_{t-1}^{2}+\delta_{0}\left(\varepsilon_{t-1}-\gamma \sigma_{t-1}\right)^{2} \\
& +\sum_{i=1}^{m-1}\left(\alpha_{i}+\beta_{i} \sigma_{t-1}^{2}+\delta_{i}\left(\varepsilon_{t-1}-\gamma \sigma_{t-1}\right)^{2}\right) G\left(\sigma_{t-1}^{2} ; \kappa_{i}\right),
\end{aligned}
$$

where $\left(\varepsilon_{t}\right)$ is a series of identically and independently distributed zero mean and unit variance random variables (strict white noise) and, for any $\kappa \in \mathbb{R}^{d}$ (transition parameters), the function $G(\cdot ; \kappa):(0, \infty) \rightarrow[0,1]$ is continuous and increasing such that $G(x ; \kappa) \rightarrow 1$ as $x \rightarrow \infty$. In addition, $G$ is also a continuous function of $\kappa$.

In contrast to other GARCH processes the conditional variance process (1) is driven directly by the innovations $\varepsilon_{t}$ and not by the error process $u_{t}$. When the number of limiting regimes is $m=1$ and the conditional mean of the log returns process $X_{t}=\log \left(S_{t}\right)-\log \left(S_{t-1}\right)$ 
is governed by $\mu_{t}=r+\lambda \sigma_{t}^{2}$, our model reduces to the $\operatorname{GARCH}(1,1)$ process proposed in Heston and Nandi (2000). This simplification of the ordinary GARCH processes allows for the existence of a closed option pricing formula. If in addition, $\alpha_{0}=\beta_{0}=0$ the resulting process $X_{t}$ coincides with the discrete time geometric Brownian motion.

The transition function $G(\cdot ; \kappa)$ may be the cumulative distribution function of any positive continuous random variable, for example, the exponential or gamma distributions. Hereafter, for convenience, the notation $G_{i, t} \equiv G\left(\sigma_{t-1}^{2} ; \kappa_{i}\right)$ is used.

In this paper, we focus our attention to the case where the conditional mean of the process is driven by

$$
\mu_{t}=\mu+\sum_{i=1}^{p} \psi_{i} X_{t-i}
$$

a linear autoregession of order $p[\operatorname{AR}(p)]$. This model can be considered as an extension of a Heston-Nandi GARCH $(1,1)$ type model where the conditional mean is driven by (2) instead of a garch-in-the-mean specification. We are interested in studying the statistical properties of multiple-regime dynamics (1) by employing the theoretical results presented in Meitz and Saikkonen (2008a) for nonlinear AR-GARCH models. The notation MRNGARCH $(p, m, 1,1)$ will be used to refer to this model, where $p$ corresponds to the order of the AR specification and $m$ the number of limiting regimes.

As a standard assumption, the vector of parameters of the $\operatorname{MRNGARCH}(p, m, 1,1)$ process

$$
\boldsymbol{\theta}=\left(\mu, \psi_{1}, \ldots, \psi_{p}, \alpha_{0}, \ldots, \alpha_{m-1}, \beta_{0}, \ldots, \beta_{m-1}, \delta_{0}, \ldots, \delta_{m-1}, \gamma, \kappa_{1}, \ldots, \kappa_{m-1}\right)^{\prime}
$$

is constrained to be in the interior of a compact and convex parameter space $\Theta \subseteq \mathbb{R}^{3 m+d(m-1)+p+2}$.

In order to derive the conditions for stationarity and the existence of finite moments of the process $\left(u_{t}\right)$, it is necessary to make the following assumptions

Assumption 1. The underlying (Lebesque) density function of $\varepsilon_{t}$ is positive and lower semi-continuous on $\mathbb{R}$. 
Assumption 2. (i) The derivatives of $G(\cdot ; \kappa)$ exist up to any order and are continuous.

(ii) For all $\kappa, \lim _{x \rightarrow-\infty} x G(x ; \kappa)=\lim _{x \rightarrow+\infty} x(1-G(x ; \kappa))=0$; if $\kappa \neq \kappa_{0}$, then for some $\bar{x}$, $G(\bar{x} ; \kappa) \neq G\left(\bar{x} ; \kappa_{0}\right)$.

(iii) For all $\kappa, \lim _{y \rightarrow-\infty} y^{2} G(y ; \kappa)=\lim _{y \rightarrow+\infty} y^{2}(1-G(y ; \kappa))=0$; if $\kappa \neq \kappa_{0}$, then for some $\bar{y}$, $G(\bar{y} ; \kappa) \neq G\left(\bar{y} ; \kappa_{0}\right)$.

Assumption 3. (i) The parameters $\alpha_{j}, \beta_{j}$, and $\delta_{j}, j=0, \ldots, m-1$ satisfy the restrictions

$$
\sum_{j=0}^{k} \alpha_{j}>0, \quad \sum_{j=0}^{k} \beta_{j} \geq 0 \text { and } \sum_{j=0}^{k} \delta_{j} \geq 0, \forall k=0, \ldots, m-1
$$

(iii) The transition distribution parameters $\kappa_{i}, i=1, \ldots, m-1$ are such that

$$
G_{1, t} \geq \cdots \geq G_{m-1, t}, \forall t
$$

Assumption 1 and 2 are fundamental for the mathematical derivations that are developed in the rest of the paper. Assumption 3 ensures strictly positive conditional variances in equation (1).

The parameters $\delta_{j}, j=0,1, \ldots, m-1$ determine the kurtosis of the distribution of $u_{t}$ and all the $\delta_{j}$ being zero implies a deterministic time varying variance. The sign of the correlation between the variance process and spot return is then determined by the parameter $\gamma$. Provided the existence of the third moment of $\varepsilon_{t}$, from Assumption 1 it can be verified that

$$
\operatorname{Cov}_{t-1}\left(\sigma_{t+1}^{2}, X_{t}\right)=-2 \gamma\left(\delta_{0}+\sum_{i=1}^{m-1} \delta_{i} G_{i, t+1}\right) \sigma_{t}^{2}
$$

From Assumption 3, it follows that $\delta_{0}+\sum_{i=1}^{m-1} \delta_{i} G_{i, t+1} \geq 0$, hence, positive values of $\gamma$ lead to negative correlation between future volatility and returns. The model is then consistent with Black (1976) postulate and the widely documented leverage effect in the literature for stock returns (see for example, Christie (1982)).

In the following proposition (see appendix for proof), necessary and sufficient conditions are stated for ensuring strict stationary $M R N G A R C H(p, m, 1,1)$ process $(1)-(2)$. 
Proposition 1. Suppose that the process $u_{t}$ defined by (1) and (2) satisfies the Assumptions 1-3.

(i) The process is stationary and ergodic with $E\left[\left|X_{t}\right|^{2}\right]<\infty$ and $E\left[\sigma_{t}^{2}\right]<\infty$ if

$$
\sum_{i=1}^{p}\left|\psi_{i}\right|<1
$$

and

$$
\mathrm{E}\left[\log \left(\beta_{0}+\gamma^{2} \delta_{0}+\sum_{i=1}^{m-1} \beta_{i}+\gamma^{2} \sum_{i=1}^{m-1} \delta_{i}+\sum_{i=1}^{m-1} \max \left\{\delta_{i}, 0\right\} \varepsilon_{t}^{2}\right)\right]<0
$$

(ii) A sufficient condition for stationary and ergodicity of $\left(u_{t}, \sigma_{t}^{2}\right)^{\prime}$ is given by

$$
\beta_{0}+\gamma^{2} \delta_{0}+\sum_{i=1}^{m-1} \beta_{i}+\gamma^{2} \sum_{i=1}^{m-1} \delta_{i}+\sum_{i=1}^{m-1} \max \left\{\delta_{i}, 0\right\}<1
$$

It is important to remark that proposition 1 is also valid in the case of a GARCH-in-themean type model

$$
\mu_{t}=\mu+\lambda \sigma_{t}^{2}
$$

Conditions for stationarity and ergodicity of the resulting process can be derived by following similar ideas to those provided in the proof of proposition 1 and the results given by Meitz and Saikkonen (2008b) .

Finally, it is interesting to highlight that the continuous-time limit of the variance process $\sigma_{t}^{2}$ of the MRNGARCH model converges weakly to a variance process, $V_{t}$ which is the squareroot process of Feller (1951), and Cox, Ingersoll Ross (1985)

$$
d V_{t}=a\left(b-V_{t}\right) d t+\sigma \sqrt{V_{t}} d W_{t}
$$

where $W_{t}$ is a Wiener process. Equation (11) motivates the possibility of exploiting the proposed model for valuing financial assets. Option pricing under this stochastic volatility framework is left for future research. 


\section{Parameter estimation and the number of regimes}

In this section we consider in detail the parameter estimation and develop a sequential procedure to tackle the problem of determining the number of regimes of the MRNGARCH model.

\subsection{Parameter estimation}

Consider $x_{1}, \ldots, x_{n}$ realizations of the process $\left(X_{t}\right)$ defined by $X_{t}=\mu_{t}+u_{t}$, where the conditional mean $\mu_{t}$ follows the dynamics (2) and the error process $\left(u_{t}\right)$ is defined by the pure MRNGARCH model (1), i.e an MRNGARCH(p,m,1,1) process. Conditional on initial realized values of $x_{-p+1}, \ldots, x_{0}$ and $\sigma_{0}$, the quasi-log-likelihood function of the model is defined as

$$
L_{n}(\boldsymbol{\theta})=\sum_{t=1}^{n} l_{t}(\boldsymbol{\theta})
$$

where $l_{t}(\boldsymbol{\theta})=-\frac{1}{2} \log (2 \pi)-\frac{1}{2} \log \left(\sigma_{t}^{2}\right)-\frac{u_{t}^{2}}{2 \sigma_{t}^{2}}$ is the log-likelihood contribution arising from the $t$ th observation.

As the underlying distribution of $x_{t}$ is unknown, (12) is treated as an objective function to be maximized (rather than a Gaussian likelihood), i.e. the vector of parameters $\boldsymbol{\theta}$ is estimated by quasi-maximum likelihood $(Q M L)$, and the estimator obtained is the $Q M L E$ of $\boldsymbol{\theta}$ and defined as

$$
\widehat{\boldsymbol{\theta}}_{n}=\underset{\boldsymbol{\theta} \in \Theta \subseteq \mathbb{R}^{3 m+d(m-1)+p+2}}{\arg \max } L_{n}(\boldsymbol{\theta}) .
$$

Two possible sources of non-identifiability of the model are the reducibility and the interchangeability of regimes. The former is related to the presence of irrelevant regimes and the second to permutations of regimes that may lead to the same values of the likelihood function. To avoid any permutation of regimes it is necessary to impose some restrictions on the parameters of the transition function $G$. In this investigation we take the transition 
function to be the Gamma distribution

$$
G(x ; \kappa)=\int_{0}^{x} \frac{s^{r}}{\Gamma(r)} u^{r-1} e^{-s u} d u
$$

with parameters $\kappa=(r, s)^{\prime}, r, s>0$. In this case, the problem of interchangeability can be prevented by imposing the restrictions ${ }^{1}$

$$
s_{1}>\cdots>s_{m-1} \text { and } r_{1} \leq \cdots \leq r_{m-1}
$$

since together imply Assumption 3(ii).

However, if there exists a regime $i \in\{1, \ldots, m-1\}$ such that $\alpha_{i}=\beta_{i}=\delta_{i}=0$, then the parameters in $r_{i}$ and $s_{i}$ remain unidentifiable so the model contains an irrelevant regime. To guarantee that there are no irrelevant regimes, the following additional assumption is required.

Assumption 4. The parameters $\alpha_{i}, \beta_{i}$ and $\delta_{i}$ do not vanish jointly for some regime $i \in\{1, \ldots, m-1\}$.

The parameter estimation strategy adopted in this article, together with an outline of the procedure is given in the following remark.

Remark 1. Consider the transformations

$$
\begin{gathered}
\alpha_{k}=e^{\alpha_{k}^{*}}-\sum_{j=0}^{k-1} \alpha_{j}, \beta_{k}=e^{\beta_{k}^{*}}-\sum_{j=0}^{k-1} \beta_{j}, \delta_{k}=e^{\delta_{k}^{*}}-\sum_{j=0}^{k-1} \delta_{j}, k=0,1, \ldots, m-1 . \\
r_{j}=r_{j-1}+e^{r_{j}^{*}}, s_{j}=s_{j-1}-e^{s_{j-1}^{*}}, j=1, \ldots, m-1,
\end{gathered}
$$

where

$$
\alpha_{0}^{*}, \ldots, \alpha_{m-1}^{*}, \beta_{0}^{*}, \ldots, \beta_{m-1}^{*}, \delta_{0}^{*}, \ldots, \delta_{m-1}^{*}, r_{1}^{*}, \ldots, r_{m-1}^{*}, s_{1}^{*}, \ldots, s_{m-1}^{*}
$$

are (unrestricted) real numbers, and the conventions $\sum_{j=0}^{-1} \equiv 0$ and $r_{0}=s_{0}$ are used. Recursive formulas (16) and (17) define an unconstrained re-parametrization of the quasi- 
log-likelihood function (12) in terms of parameters (18) and $\mu, \psi_{1}, \ldots, \psi_{p}, \gamma$. The resulting optimization problem can be solved through unconstrained optimization methods. To estimate the initial values for the parameters, we employ a method based on a genetic algorithm. The optimization procedure consists of a sequential application of the algorithm of Berndt, B.H. Hall, R.E. Hall and Hausman (1974) (BHHH) in combination with a method of Mebane and Sekhon (2011) that combines evolutionary search algorithms with derivative-based (Newton or quasi-Newton) methods.

The transformations considered in Remark 1 are useful in order to tackle the problem of maximizing the function (12) in terms of its parameters in order to obtain good $Q M L E$ estimates.

\subsection{Estimation of the number of regimes}

In Definition 1 the number of limiting regimes $m$ in the MRNGARCH model is not known in advance. To address this problem, we follow a "specific-to-general" modeling strategy in a similar fashion to Medeiros and Veiga (2009). Consider the model MRNGARCH $(p, m, 1,1)$ defined by (1) and (2) with $m$ limiting regimes and the possibility of adding an extra regime represented by $\left(\alpha_{m}+\beta_{m} \sigma_{t-1}^{2}+\delta_{m}\left(\varepsilon_{t-1}-\gamma \sigma_{t-1}\right)^{2}\right) G_{m, t}$. Since $G_{m, t}=0$ for any $\sigma_{t-1}^{2}, r_{m}>0$ if the scale parameter $s_{m}$ is zero, an appropriate null hypothesis is

$$
H_{0}: s_{m}=0,
$$

against the alternative $H_{1}: s_{m}>0$. As the base model of $m$ regimes is not identified under $H_{0}$, following Luukkonen, Saikkonen and Teräsvirta (1988), it is convenient to expand $G$ about the null $s_{m}=0$. For this purpose, we use a novel result on the approximation to the so-called incomplete gamma function. A direct application of Lemma 3 (see appendix) to 
the Gamma distribution (14) around $s=0$ leads to the approximation,

$$
G(x ; r, s)=\frac{c_{r}}{\Gamma(r)} s^{k} x^{2}+R_{1}
$$

valid for all $x<M$ and any $M>0$, where $R_{1}$ is the remainder ${ }^{2}$ and $k=k(r, s, M)>0$. By using the expansion (20) for $G_{m, t}$, the conditional variance can be rewritten as

$$
\begin{aligned}
\sigma_{t}^{2} & =\alpha_{0}+\beta_{0} \sigma_{t-1}^{2}+\delta_{0}\left(\varepsilon_{t-1}-\gamma \sigma_{t-1}\right)^{2}+\sum_{i=1}^{m-1}\left(\alpha_{i}+\beta_{i} \sigma_{t-1}^{2}+\delta_{i}\left(\varepsilon_{t-1}-\gamma \sigma_{t-1}\right)^{2}\right) G_{i, t} \\
& +a_{1} \sigma_{t-1}^{4}+a_{2} \sigma_{t-1}^{6}+a_{3}\left(\varepsilon_{t-1}-\gamma \sigma_{t-1}\right)^{2} \sigma_{t-1}^{4}+R_{2}
\end{aligned}
$$

where $a_{1}=b \alpha_{m}, a_{2}=b \beta_{m}, a_{3}=b \delta_{m}$, with $b=c_{r} \Gamma\left(r_{m}\right)^{-1} s_{m}^{k}$ and $k=k\left(r_{m}, s_{m}, M\right), M$ is any upper bound for the series $\left(\sigma_{t-1}^{2}\right)_{t=1}^{n}$, and the remainder $R_{2}$ vanishes under $H_{0}$.

When a new regime is considered in the model, the vector of parameters is given by $\boldsymbol{\vartheta}=\left(\boldsymbol{\theta}^{\prime}, \mathbf{a}^{\prime}\right)^{\prime}$ where $\mathbf{a}=\left(a_{1}, a_{2}, a_{3}\right)^{\prime}$. It is straightforward to verify that the corresponding score vector can be written as

$$
\begin{aligned}
S(\boldsymbol{\vartheta}) & \equiv \sum_{t=1}^{n} \frac{\partial l_{t}(\boldsymbol{\vartheta})}{\partial \boldsymbol{\vartheta}}=\sum_{t=1}^{n} u_{t}\left[\frac{1}{\sigma_{t}^{2}} \frac{\partial \mu_{t}}{\partial \boldsymbol{\vartheta}}\right]+\sum_{t=1}^{n}\left(u_{t}^{2}-\sigma_{t}^{2}\right)\left[\frac{1}{2 \sigma_{t}^{4}} \frac{\partial \sigma_{t}^{2}}{\partial \boldsymbol{\vartheta}}\right] \\
& =\sum_{t=1}^{n}\left[\frac{\partial \mu_{t}}{\partial \boldsymbol{\vartheta}}: \frac{\partial \sigma_{t}^{2}}{\partial \boldsymbol{\vartheta}}\right] W_{t}\left[\begin{array}{c}
u_{t} \\
u_{t}^{2}-\sigma_{t}^{2}
\end{array}\right]=\sum_{t=1}^{n} \Upsilon_{t}^{\prime} W_{t} \psi_{t}
\end{aligned}
$$

where

$$
\Upsilon_{t}^{\prime} \equiv\left[\frac{\partial \mu_{t}}{\partial \boldsymbol{\vartheta}}: \frac{\partial \sigma_{t}^{2}}{\partial \boldsymbol{\vartheta}}\right], W_{t} \equiv\left[\begin{array}{cc}
\frac{1}{\sigma_{t}^{2}} & 0 \\
0 & \frac{1}{2 \sigma_{t}^{4}}
\end{array}\right], \text { and } \psi_{t} \equiv\left[\begin{array}{c}
u_{t} \\
u_{t}^{2}-\sigma_{t}^{2}
\end{array}\right]
$$

Here, triple dots $\vdots$ are used as a column matrix block separator. The Fisher information matrix is then calculated as

$$
J(\boldsymbol{\vartheta}) \equiv-\mathrm{E}\left(\frac{\partial^{2} l_{t}(\boldsymbol{\vartheta})}{\partial \boldsymbol{\vartheta} \partial \boldsymbol{\vartheta}^{\prime}}\right)=\mathrm{E}\left(\frac{1}{\sigma_{t}^{2}} \frac{\partial \mu_{t}}{\partial \boldsymbol{\vartheta}} \frac{\partial \mu_{t}}{\partial \boldsymbol{\vartheta}^{\prime}}\right)+\frac{1}{2} \mathrm{E}\left(\frac{1}{\sigma_{t}^{4}} \frac{\partial \sigma_{t}^{2}}{\partial \boldsymbol{\vartheta}} \frac{\partial \sigma_{t}^{2}}{\partial \boldsymbol{\vartheta}^{\prime}}\right)=\mathrm{E}\left(\Upsilon_{t}^{\prime} W_{t} \Upsilon_{t}\right)
$$

by applying the law of iterated expectations and conditioning with respect to $\Im_{t-1}$ and noting 
that from the recursive expression $(A .7)$ in the Appendix, the partial derivative $\frac{\partial \mu_{t}}{\partial \vartheta}$ is $\Im_{t-1}$ measurable, as is $\frac{\partial^{2} \mu_{t}}{\partial \vartheta \partial \vartheta^{\prime}}$.

Under the null hypothesis (19), it is implied that $a_{1}=a_{2}=a_{3}=0$ so that expressions (A.14), (A.15) and (A.16) for vector $\mathbf{V m}_{t}$ and scalars $K m_{t}$ and $L m_{t}$, respectively, vanish. Then formulas (A.8) and (A.9) in Lemma 2 from the Appendix, corresponding to the partial derivatives of the local approximation (21), are simplified as

$$
\begin{aligned}
\frac{\partial \sigma_{t}^{2}}{\partial \boldsymbol{\theta}} & =\mathbf{V}_{t}+K_{t} \frac{\partial \sigma_{t-1}^{2}}{\partial \boldsymbol{\theta}}-L_{t} \frac{\partial \mu_{t-1}}{\partial \boldsymbol{\theta}} \\
\frac{\partial \sigma_{t}^{2}}{\partial \mathbf{a}} & =\mathbf{V} \mathbf{a}_{t}+K_{t} \frac{\partial \sigma_{t-1}^{2}}{\partial \mathbf{a}}, \\
\frac{\partial \mu_{t}}{\partial \boldsymbol{\theta}} & =\mathbf{E}_{t}-\sum_{i=1}^{p} \phi_{i} \frac{\partial \mu_{t-i}}{\partial \boldsymbol{\theta}},
\end{aligned}
$$

where $\mathbf{V}_{t}, \mathbf{V} \mathbf{a}_{t}, K_{t}$ and $L_{t}$ are given by $(A .10)$, (A.17), (A.11) and (A.12), respectively. Hence, by noting that $\frac{\partial \sigma_{0}^{2}}{\partial \vartheta}=\frac{\partial \mu_{\tau}}{\partial \vartheta}=0, \tau=-p+1, \ldots, 0$, the recursive forms (24) and (25) have solutions

$$
\begin{aligned}
\frac{\partial \sigma_{t}^{2}}{\partial \boldsymbol{\theta}} & =\mathbf{V}_{t}+\sum_{i=1}^{t-1} \prod_{j=i+1}^{t} K_{j} \mathbf{V}_{i}-\sum_{i=1}^{t-1} \prod_{j=i+2}^{t} K_{j} L_{i+1} \frac{\partial \mu_{i}}{\partial \boldsymbol{\theta}}, \\
\frac{\partial \sigma_{t}^{2}}{\partial \mathbf{a}} & =\mathbf{V} \mathbf{a}_{t}+\sum_{i=1}^{t-1} \prod_{j=i+1}^{t} K_{j} \mathbf{V} \mathbf{a}_{i}, \\
\frac{\partial \mu_{t}}{\partial \boldsymbol{\theta}} & =\mathbf{E}_{t}+\sum_{j=1}^{t-1} \sum_{k=1}^{j} D_{j k} \mathbf{E}_{j},
\end{aligned}
$$

where $D_{j k}=(-1)^{k} \sum_{A} \phi_{i_{1}} \cdots \phi_{i_{k}}$ with the subset $A$ formed by indexes such that $1 \leq$ $i_{1}, \ldots, i_{k} \leq q$ and $i_{1}+\cdots+i_{k}=j$.

A remaining aspect not addressed in this document is the validity, under the null, of $n^{-1 / 2} S(\boldsymbol{\vartheta}) \stackrel{d}{\rightarrow} N(0, J(\boldsymbol{\vartheta}))$, where $J(\boldsymbol{\vartheta})$ can be estimated by $J_{n}(\boldsymbol{\vartheta})=\frac{1}{n} \sum_{t=1}^{n} \widehat{\Upsilon}_{t}^{\prime} \widehat{W}_{t} \widehat{\Upsilon}_{t}$, where "^" denotes that each function is evaluated at $\widehat{\boldsymbol{\theta}}_{n}$. In this case, under the null hypothesis 
(19), the $L M$ statistic is then given by

$$
L M=n \sum_{t=1}^{n} \widehat{\Upsilon}_{t}^{\prime} \widehat{W}_{t}\left[\begin{array}{c}
\widehat{u}_{t} \\
\widehat{u}_{t}^{2}-\widehat{\sigma}_{t}^{2}
\end{array}\right]\left[\sum_{t=1}^{n} \widehat{\Upsilon}_{t}^{\prime} \widehat{W}_{t} \widehat{\Upsilon}_{t}\right]^{-1} \sum_{t=1}^{n} \widehat{\Upsilon}_{t}^{\prime} \widehat{W}_{t}\left[\begin{array}{c}
\widehat{u}_{t} \\
\widehat{u}_{t}^{2}-\widehat{\sigma}_{t}^{2}
\end{array}\right]
$$

The test statistic (30) can be constructed under the normality assumption. However as the investigation of normality is not considered in this article we instead implement a robust version of the $L M$ test against general error distribution by a direct application of Theorem 2.1 in Wooldridge (1990). For the purpose of this paper, consider the estimated gradients $(24)$ and $(26)$ in the $(3 m+d(m-1)+p+2) \times 2$ matrix

$$
\widehat{\Upsilon}_{t}^{\prime}=\left[\frac{\partial \mu_{t}\left(\widehat{\boldsymbol{\theta}}_{n}\right)}{\partial \boldsymbol{\theta}}: \frac{\partial \sigma_{t}^{2}\left(\widehat{\boldsymbol{\theta}}_{n}\right)}{\partial \boldsymbol{\theta}}\right]
$$

which corresponds to the transpose of $-E\left(\frac{\partial_{t} \psi\left(\widehat{\boldsymbol{\theta}}_{n}\right)}{\partial \boldsymbol{\theta}} \mid X_{t}\right)$. Define the matrix of weights

$$
\widehat{W}_{t}^{1 / 2}=\left[\begin{array}{cc}
1 / \widehat{\sigma}_{t} & 0 \\
0 & 1 /\left(\sqrt{2} \widehat{\sigma}_{t}^{2}\right)
\end{array}\right]
$$

as well as the auxiliary matrices

$$
\widetilde{\Upsilon}_{t} \equiv \widehat{W}_{t}^{1 / 2} \widehat{\Upsilon}_{t}, \widetilde{\Psi}_{t} \equiv \widehat{W}_{t}^{1 / 2} \widehat{\Psi}_{t} \text { and } \widetilde{\psi}_{t} \equiv \widehat{W}_{t}^{1 / 2}\left[\begin{array}{c}
\widehat{u}_{t} \\
\widehat{u}_{t}^{2}-\widehat{\sigma}_{t}^{2}
\end{array}\right]
$$

where $\widehat{\varepsilon}_{t}=\frac{\widehat{u}_{t}}{\widehat{\sigma}_{t}}$ are the standardized residuals. The procedure for performing the $L M$ test in regression stages under non-normality can be stated as follows.

1. Compute $\widehat{\sigma}_{t}^{2}$, the estimated conditional variance of the process $\left(X_{t}\right)$ driven by equations (2) and (1) under the null, and the gradient estimates $\widehat{\Upsilon}_{t}$ from the formulas (27), (28) and (29) where $\mathbf{V}_{t}, \mathbf{V} \mathbf{a}_{t}, \mathbf{E}_{t}, K_{t}, L_{t}, t=1, \ldots, n$ are obtained by $(A .10),(A .17),(A .13),(A .11)$ and (A.12).

2. Regress $\widetilde{\Upsilon}_{t}$ on $\widetilde{\Psi}_{t}$ and compute the residual vectors $\widehat{\mathbf{r}}_{t}, t=1, \ldots, n$. 
3. Regress 1 on $\widetilde{\psi}_{t}^{\prime} \widehat{\mathbf{r}}_{t}$ and compute the residual sum of squares $S S R$. The test statistic

$$
L M^{R}=n-S S R
$$

is asymptotically distributed $\chi^{2}$ with 3 degrees of freedom.

Despite the robust version of the $L M$ test it is preferred to the non-robust tests (see Lundbergh and Teräsvirta, 2002), possible outliers in the data may affect the results of the sequence of $L M$ test. However, following van Dijk, Franses and Lucas (1999) it is also possible to develop an outlier-robust version of the $L M$ test developed in this work.

For practical purposes in empirical applications, we follow Medeiros and Veiga (2009) and implement a sequential application of the proposed LM test. First, linearity is tested against an $\operatorname{ARCH}(q)$ model at significance level $\varpi$ using the Engle (1982) test. If the null hypothesis of homoskedasticity is rejected, a MRNGARCH$(1,1,1,1)$ model is estimated and tested against an MRNGARCH model with two limiting regimes at the significance level $\varpi v$ for a reduction parameter $0<v<1$. The procedure continues recursively by estimating an MRNGARCH$(1, m, 1,1)$ model with $m$ limiting regimes and testing it against the version with $m+1$ limiting regimes. The sequential process stops when the null hypothesis (19) can not be rejected at the significance level $\varpi v^{m}$. At each step, the significance level is reduced and converges to zero as the number of limiting regimes increases so that models with a large number of parameters are less likely to be chosen.

\section{Empirical Application}

In this section an empirical application of the MRNGARCH model is implemented to evaluate its ability in forecasting volatility in the context of the major world stock indexes and the corresponding exchange rates. The exercise is based on a data set of 18 stock indexes and their corresponding foreign exchange (FX) rates to U.S. dollar. The series are chosen to represent some of the most important financial markets. The sample period is divided into two 
parts. The first for in-sample analysis, and the second to test the forecasting performance of the models. The in-sample period spans from September 3, 1996 to August 29, 2008 and the out-of-sample spans from September 1, 2008 to June 30, 2010. The in-sample consists of 3,129 daily observations, while the out-of-sample consists of 478 daily observations. Our study is based on a data set of daily closing prices obtained from Thomson Reuters DataStream ${ }^{3}$.

Table 1 shows, for each stock index and its corresponding FX currency rate to USD, some descriptive statistics for daily log-returns of the 31 series considered in the analysis. The sample mean (mean), sample standard deviation (std), sample skewness $(s k)$ and sample kurtosis $(k u)$, in excess to three, are included.

[Table 1 around here]

For each series we estimate the conditional variance via the standard $\operatorname{GARCH}(1,1)$ model and also the $\operatorname{EGARCH}(1,1)$, GJR $(1,1)$ and $\operatorname{APARCH}(1,1)$ specifications to allow for leverage effects. For the conditional mean a simple $\operatorname{AR}(1)$ is considered. Tables 2, 3, 4 and 5 contain the estimation results and the diagnostic tests for each of the types of GARCH models employed. The numbers in parenthesis below the parameter estimates are their robust standard errors. The basic statistics $s t d, s k$ and $k u$ are computed for standardized residuals. To test the adequacy of these models additional diagnostic tests are implemented. We compute the Ljung-Box statistics to test the strict white noise hypothesis to the raw, squared and absolute standardized residuals; the corresponding p-values are denoted by columns $L j B 1$, $L j B 2$ and $L j B A b s$. For testing asymmetry, we report the p-values of the sign bias $(S b)$, negative size bias $(N s b)$, positive size bias $(P s b)$, and joint $(J s b)$ tests proposed by Engle and $\mathrm{Ng}$ (1993). In addition, for evaluating interval forecast estimations, we implement the unconditional coverage, independence, and conditional coverage tests proposed by Christoffersen (1998). By considering a 95\% confidence interval, the corresponding p-values of these tests are presented in columns $P c, P i$ and $P c c$. Finally, Bayesian information criterion $(B I C)$ and un-normalized log-likelihood values (Likelihood) are reported. 
For all the series, the coefficients are statistically significant at the $5 \%$ level. The normalized residuals have close to zero skewness coefficients, particularly negative in the case of equities. On the other hand, normalized residuals from simple GARCH and EGARCH specifications have, on average, slightly lager kurtosis to those obtained from the GJR and APARCH alternatives. When comparing the values of BIC and likelihood, a similar situation occurs among models, where APARCH specification has the smallest BIC level and the highest likelihood ones. The sum of corresponding estimated coefficients reveals a high persistence level in the simple GARCH model (over 0.94, and, on average, close to 1); except for two series, the situation is similar for the EGARCH model (17 series with levels over 1, inclusive). In the case of the GJR model, most of the series present an explosive regime, as the sum of $\beta_{0}, \delta_{0}$ and $\gamma_{0}$ is greater than one for most of the equity series and about half of the FX rates; meanwhile, the APARCH model has relatively moderate persistence for most of the series.

By inspection of the sign bias, negative sign bias, positive sign bias, and joint tests pvalues columns, there are asymmetric effects in the standardized residuals from the simple GARCH model as well as, though to a lesser degree, for the other three alternative models. In about half of the series considered there are some asymmetric effects, at the $5 \%$ significance level.

With regards to the analysis of coverage tests, apart from five equity series (IBEX35 and DAX30 -GARCH-, SMI -EGARCH-, ALSI -GJR- and IBOVESPA -APARCH model-), all specifications produce the correct confidence intervals for the series.

[Tables 2, 3, 4 and 5 around here]

We now consider the MRNGARCH $(1,1,1,1)$ specification as the basis model. We proceed to determine the number of limiting regimes by implementing the robust version of the LM test that was developed in the previous section. An initial significance level of $5 \%$ was used for the sequence of LM tests and halved at each step $(v=0.5)$. The estimation is performed by the QML method using evolutionary search algorithms with derivative-based numerical 
optimization algorithms. Table 6 reports the parameter estimates. We follow, for similar reasons, Silvennoinen and Terasvirta (2009) and report p-values and standard errors in this empirical analysis under the assumption of asymptotic normality of the $Q M L E$.

The main findings indicate that equity indices tend to allow for multiple regimes more often in comparison to FX rates. In all but five equity series (AEX, ATX, SET, SMI and TSX), the null hypothesis of no additional regime is rejected. The tests show evidence of two limiting regimes for ten series: ALSI, ASX200, BSE100, CAC40, FTSE100, IBEX35, IBOVESPA, ISE100, NIKKEI225 and RTSI. For the DAX30, IPC and S\&P500 three limiting regimes are found. In contrast, only three of the FX series considered, namely, AUD, MXN and RUB, showed evidence of additional limiting regimes.

It is important to mention that for all equity indices the parameter $\gamma$ is positive and statistically different from zero. As discussed in the previous section, this finding implies that negative movements of the returns tend to have a larger impact on volatility rather than positive ones. In contrast, for the FX series, apart from CHF and JPY, the sign of the leverage parameter is negative. However, the parameter $\gamma$ is is not statistically significantly different from zero for all FX series.

The diagnostic test results of the model are tabulated in Table 7 . When comparing the $L j B 1, L j B 2$ and $L j B A b s$ values to those obtained via the alternative GARCH specifications, it can be observed that the white noise assumption for standardized residuals is satisfied for more series for the MRNGARCH model. In addition, the interval coverage tests, MRNGARCH outperforms the other alternatives since some of the reported interval coverage tests p-values are below the $5 \%$ confidence level for only one of the equity indexes (S\&P500) and four exchange rates (GBP, INR, RUB, TRY). Moreover, the Sb, Nsb, Psb and $J s b$ columns indicate that MRNGARCH fails to adequately describe the asymmetric relation between returns and volatility in only three series, namely, the FX rates BRL, CAD and JPY.

[Tables 6 and 7 around here] 
When comparing the Likelihood values to the GARCH model alternatives, it can be noted that, with the sole exception of the CAD index, MRNGARCH model outperforms the other alternatives. Meanwhile, apart from the NIKKEI, RTSI, THB and ZAR series, the BIC values lead to the same conclusion. Where the MRNGARCH model does not outperform, the GJR and APARCH specifications seem to be acceptable models.

The final step in the empirical study addresses the forecasting performance evaluation of the models. Following Hansen and Lunde (2005), we use the following three sample expected loss functions (criterion) in our empirical analysis

$$
M A E \equiv n^{-1} \sum_{t=1}^{n}\left|\sigma_{t}^{2}-h_{t}\right|, M S E_{1} \equiv n^{-1} \sum_{t=1}^{n}\left(\sigma_{t}^{2}-h_{t}\right)^{2}, M S E_{2} \equiv n^{-1} \sum_{t=1}^{n}\left(\sigma_{t}^{4}-h_{t}^{2}\right)^{2},
$$

where $\left(h_{t}\right)$ is a forecast of the underlying conditional variance process $\left(\sigma_{t}^{2}\right)$. As a proxy of the variance, the squared returns are used in the analysis and the volatility forecast considers a one-day-ahead horizon. In Table 8 we present the values of each error criteria for the MRNGARCH model and the GARCH, EGARCH, GJR and APARCH alternatives. For each series the minimum values are highlighted in bold. The main finding is that MRNGARCH model outperforms the alternative specifications. The MRNGARCH model produces the lowest $M A E$ values for 16 out of the 18 equity indices (89\%) and 10 out of the $13 \mathrm{FX}$ series (77\%). Under this criteria, EGARCH, GJR and APARCH specifications are also acceptable, or with similar $M A E$ values inclusive, in 3, 8 and 9 cases, respectively. On the other hand, $M S E_{1}$ figures indicate that MRNGARCH is the best model in 16 out of the 18 equity indices (89\%) and 8 out of $13 \mathrm{FX}$ series $(62 \%)$ which is similar to the results for the $M S E_{2}$ criteria.

[Table 8]

In order to statistically discriminate if a particular forecasting model is outperformed by alternative forecasts we implement the so called superior predictive ability (SPA) test proposed by Hansen (2005). The null hypothesis of the SPA test is that a given model is 
not inferior to any other competing models in terms of their expected loss. The numbers in parenthesis of Table 8 are the p-values of the SPA test. The main remark is that the simple GARCH is the model with the most rejections by the SPA test for all the implemented loss criterions ( $88 \%$ rejections on average), in contrast, the proposed MRNGARCH is the model with the highest forecasting effectiveness (26\% rejections on average). When losses are measured through the $M A E$ criterion, MRNGARCH model produces satisfactory forecasts for 15 out of the 18 equity indices (83\%) and 11 out of the 13 FX series (85\%). In comparison to their performance on equity series, the APARCH, GJR and EGARCH models are more appropriate for forecasting FX volatilities: $44 \%, 56 \%$ and $28 \%$ for the equity indexes, respectively, in contrast to the corresponding $85 \%, 62 \%$ and $62 \%$ ratios observed in FX rates.

When the $M S E_{1}$ is used, the MRNGARCH model is again the best performer: adequate forecasts for 26 out of the 31 series (71\%) against the $45 \%, 39 \%$ and $39 \%$ observed for APARCH, GJR and EGARCH alternatives, respectively. A similar situation is observed for the $M S E_{2}$ criterion. Finally, when the three criteria numbers are averaged, the MRNGARCH model shows adequate forecasts for $74 \%$ of the series, against $48 \%, 47 \%, 40 \%$ and $12 \%$ obtained for APARCH, GJR, EGARCH and simple GARCH alternatives, respectively. However, these four alternatives shows better forecasting performance for FX rates in comparison to equity series.

\section{Conclusions}

In this paper we propose a new nonlinear $\mathrm{GARCH}$ model, the multiple regime nonlinear GARCH (MRNGARCH), to describe the asymmetric behavior of financial volatility and returns and their intermittent dynamics. The model is a generalization of the HestonNandi GARCH(1,1) specification that allows multiple regimes in the conditional variance dynamics. Conditions for strict stationarity, ergodicity and existence of moments for the 
proposed model were established. Estimation of the parameters was addressed through the quasi-maximum likelihood approach and the asymptotic properties of the estimator were discussed. The number of regimes in the model was effectively determined through a sequential implementation of a procedure based on a robust Lagrange Multiplier (LM)-type specification test. To avoid the estimation of unidentified regimes, a novel expansion formula for the lower incomplete gamma function was developed.

An empirical application was implemented on a set of 18 of the major world stock indexes and their corresponding 13 foreign exchange rates to US dollar. A total of thirteen series, ten of them stock indexes, showed evidence of two limiting regimes, and three stock indexes allowed for three limiting regimes. With the sole exception of one index, the first limiting regime was associated with negative shocks, representing "bad news". Most of the stock series have two or three regimes and show a strong leverage effect while FX rates have no more than two limiting regimes and no leverage effect was detected. The forecasting performance of the MRNGARCH outperforms the GARCH, EGARCH, GJR and APARCH alternatives for the majority of the time series.

Finally the MRNGARCH model has as a continuous time limit a CIR process. This provides a framework for extending the Heston-Nandi option pricing methodology to the important case of multiple regimes. This will be the focus of a future investigation.

\section{Appendix}

\section{Proof of proposition 1}

In order to prove proposition 1 we require the following Lemma in conjunction with results obtained by Meitz and Saikkonen (2008a) [MS].

Lemma 1. The conditional variance process (1) is defined by a recursive equation

$$
\sigma_{t}^{2}=g\left(\varepsilon_{t-1}, \sigma_{t-1}^{2}\right),
$$


where $g: \mathbb{R} \times \mathbb{R}_{+} \rightarrow \mathbb{R}_{+}$satisfies $g(\varepsilon, x) \leq\left(a+b \varepsilon^{2}\right) x+c+\psi(\varepsilon)$, for all $x>0$, with $a=\beta_{0}+\gamma^{2} \delta_{0}+\sum_{i=1}^{m-1}\left(\beta_{i}+\gamma^{2} \delta_{i}\right), b=\delta_{0}+\sum_{i=1}^{m-1} \max \left\{\delta_{i}, 0\right\}, \psi(\varepsilon)=b\left(|\gamma|+\sqrt{\varepsilon^{2}+\gamma^{2}}\right)^{2}$, and some positive $c$.

\section{Proof}

From (1) we can define, for any $x>0$,

$$
\begin{aligned}
g(\varepsilon, x)= & a x+\sum_{i=1}^{m-1}\left(\beta_{i}+\gamma^{2} \delta_{i}\right)\left(G_{i}(x)-1\right) x+\alpha_{0}+\sum_{i=1}^{m-1} \alpha_{i} G_{i}(x) \\
& +\left(\delta_{0}+\sum_{i=1}^{m-1} \delta_{i} G_{i}(x)\right)\left(\varepsilon^{2}-2 \gamma x^{1 / 2} \varepsilon\right) .
\end{aligned}
$$

Note that for any $M>0$,

$$
\begin{aligned}
\sum_{i=1}^{m-1}\left(\beta_{i}+\delta_{i} \gamma^{2}\right)\left(G_{i}(x)-1\right) x & \leq \sum_{i=1}^{m-1}\left|\beta_{i}+\delta_{i} \gamma^{2}\right|\left(1-G_{i}(x)\right) x\left(1_{\{x \leq M\}}+1_{\{x>M\}}\right) \\
& \leq \sum_{i=1}^{m-1}\left|\beta_{i}+\delta_{i} \gamma^{2}\right|\left(M+\left(1-G_{i}(x)\right) x 1_{\{x>M\}}\right) .
\end{aligned}
$$

By Assumption $2(i i)$, it follows that $\left(1-G\left(x ; \kappa_{i}\right)\right) x \rightarrow 0$, as $x \rightarrow \infty$. Then by choosing $M$ sufficient large, it follows that

$$
\sum_{i=1}^{m-1}\left(\beta_{i}+\delta_{i} \gamma^{2}\right)\left(G_{i}(x)-1\right) x \leq 2 M \sum_{i=1}^{m-1}\left|\beta_{i}+\delta_{i} \gamma^{2}\right|
$$

Let $\varepsilon \neq 0$ and consider the functions $\xi(x)=\varepsilon^{2}+2|\gamma \varepsilon| x^{1 / 2}$ and $v(x)=\varepsilon^{2} x$, both defined for $x>0$. It can easily be verified that $\xi(x)=v(x)$ for the level $x=\varepsilon^{-2}\left(|\gamma|+\sqrt{\varepsilon^{2}+\gamma^{2}}\right)^{2}$, so that $\xi(x) \leq \max \left\{\left(|\gamma|+\sqrt{\varepsilon^{2}+\gamma^{2}}\right)^{2}, \varepsilon^{2} x\right\}$. This inequality is useful to guarantee that for any $\varepsilon \in \mathbb{R}$

$$
\varepsilon^{2}-2 \gamma x^{1 / 2} \varepsilon \leq \varepsilon^{2} x+\left(|\gamma|+\sqrt{\varepsilon^{2}+\gamma^{2}}\right)^{2} .
$$

By noting that $0 \leq G\left(x ; \kappa_{i}\right) \leq 1$ and combining $(A .3)$ and $(A .4)$ it follows

$$
g(\varepsilon, x) \leq\left(a+b \varepsilon^{2}\right) x+c+\psi(\varepsilon)
$$


where $c=2 M \sum_{i=1}^{m-1}\left|\beta_{i}+\delta_{i} \gamma^{2}\right|+\alpha_{0}+\sum_{i=1}^{m-1} \max \left\{\alpha_{i}, 0\right\}>0$.

From Assumptions 2(i) and 3, we observe that function $g$ defined by $(A .2)$ is smooth, strictly positive and satisfies, for any $x>0$, the limit behavior $g(\varepsilon, x) \rightarrow \infty$, as $\varepsilon \rightarrow \infty$. In other words, Assumptions $4(a)$ and $4(b)$ from MS are satisfied.

Note that by Lemma $1, g(0, x) \leq a x+c+4 b \gamma^{2}$. Then for large enough $x, g(0, x) \leq$ $(a+\epsilon) x$ for some $\epsilon>0$ such that $a+\epsilon<1$. From the smoothness and strictly positive variance property, the function $g(0, x)$ has a maximal fixed point $h^{*}$ such that $g\left(0, h^{*}\right)=h^{*}$ and $g(0, x)<x$ for all $x>h^{*}$. Suppose that for any $h_{0} \geq h^{*}$ the sequence $\left\{h_{k}=g\left(0, h_{k-1}\right)\right\}$ converges to $h^{*}$. It implies $h_{k}=g\left(0, h_{k-1}\right)<h_{k-1}$ for any $k \geq 1$, so that the sequence $\left\{h_{k}\right\}$ is non-increasing and bounded from above by $h^{*}$. Then there is $h_{1}>h^{*}$ such that $h_{k} \rightarrow h_{1}$ as $k \rightarrow \infty$. On the other hand, by the continuity of $g(0, \cdot), g\left(0, h_{k}\right) \rightarrow g\left(0, h_{1}\right)$. Thus we must have $g\left(0, h_{1}\right)=h_{1}$, and since $h^{*}$ is the maximal fixed point, $h_{1}=h^{*}$. This proofs the validity of Assumption $4(c)$ of MS. Meanwhile $(A .5)$ corresponds to their Assumption $4(d)$.

In addition, since $E\left[\varepsilon_{t}^{2}\right]=1$, assumption 1 implies that Assumption 1 of MS holds with the value $r=1$. The $A R(p)$ form in the conditional mean satisfies Assumption 2 in MS, whereas condition (7) corresponds to that of Lemma 1 ( $i i)$ of MS so that their Assumption 3 is also satisfied. By following the same arguments as in MS, the validity of their Assumption 6 can be verified.

Finally, our Assumptions 2 and 3 together with (8) imply that the process $u_{t}$ defined by (1) satisfies the necessary conditions for the validity of Theorem 1 . In order to verify last affirmation, note that from $(A .1)$ and $(A .5)$ we have that

$$
\sigma_{t-m+k+1}^{2} \leq c_{t-m+k} \sigma_{t-m+k}^{2}+c+\psi_{t-m+k}
$$

with the notation $c_{s}=a+b \varepsilon_{s}^{2}$ and $\psi_{s}=b\left(|\gamma|+\sqrt{\varepsilon_{s}^{2}+\gamma^{2}}\right)^{2}$. By iterating $k-1$ times in 
equation $(A .6)$ we obtain

$\sigma_{t-m+k+1}^{2} \leq \prod_{j=1}^{k} c_{t-m+j} \sigma_{t-m+1}^{2}+c\left(1+\sum_{j=0}^{k-2} \prod_{i=0}^{j} c_{t-m+k-i}\right)+\left(\psi_{t-m+k}+\sum_{j=0}^{k-2} \psi_{t-m+k-j-1} \prod_{i=0}^{j} c_{t-m+k-i}\right)$

If instead of $(8)$ we assume $\delta \equiv E(c)<1$. Furthermore, by noting that $\psi(\varepsilon) \leq 2 \gamma^{2}+$ $2 \gamma \max \{|\varepsilon|, \gamma\}+\varepsilon^{2}$, we denote $\eta \equiv E\left[\psi\left(\varepsilon_{t}\right)\right]<\infty$ and define $d=(c+\eta) /(1-\delta)$. By the independence of the processes $c_{t}$ and $\psi_{t}$, as well as individually,

$E\left[h\left(Z_{t-m+k}\right) \mid Z_{t-m}=z\right] \leq h(z) \delta^{k}+c\left(1+\sum_{j=0}^{k-2} \delta^{j+1}\right)+\eta\left(1+\sum_{j=0}^{k-2} \delta^{j+1}\right) \leq h(z) \delta^{k}+d$,

where $Z_{t}=\left[x_{t-1} \cdots x_{t-p} \sigma_{t}^{2}\right]$ denotes the Markov chain defined in MS and the explicit representation $h\left(Z_{t-1}\right)=g\left(u_{t-1}, \sigma_{t-1}^{2}\right)$. By following the same lines as in MS, the results in their Lemma 5 are valid for our model. Finally, it can be verified the validity of Theorem 1 of MS since the proofs for Lemmas 2, 3, 4 and 6 are also applicable to our model. As pointed out in MS, the result is still valid by just replacing $r$ by an unknown $r_{0} \in(0, r)$, so that if (8) is assumed, their Theorem 1 is valid and then our process $\left(u_{t}, \sigma_{t}^{2}\right)^{\prime}$ is stationary and ergodic. The sufficient condition $(i i)$ is consequence of this remark.

Lemma 2. (i) The gradient, with respect to the vector of parameters $\boldsymbol{\theta}$ (3), for the conditional variance of the process $\left(X_{t}\right)$ defined by (2) and (1) is given by the recursive forms

$$
\frac{\partial \sigma_{t}^{2}}{\partial \boldsymbol{\theta}}=\mathbf{V}_{t}+K_{t} \frac{\partial \sigma_{t-1}^{2}}{\partial \boldsymbol{\theta}}-L_{t} \frac{\partial \mu_{t-1}}{\partial \boldsymbol{\theta}}, \frac{\partial \mu_{t}}{\partial \boldsymbol{\theta}}=\mathbf{E}_{t}
$$

for some vectors $V_{t}, E_{t} \in \mathbb{R}^{5 m+p}$ and scalars $K_{t}$ and $L_{t}$.

(ii) The gradient with respect to the parameters $\vartheta=\left(\boldsymbol{\theta}^{\prime}, \mathbf{a}^{\prime}\right)^{\prime}$ in the local approximation (21) is given by equations

$$
\begin{aligned}
\frac{\partial \sigma_{t}^{2}}{\partial \boldsymbol{\theta}} & =\mathbf{V}_{t}+\mathbf{V m}_{t}+\left(K_{t}+K m_{t}\right) \frac{\partial \sigma_{t-1}^{2}}{\partial \boldsymbol{\theta}}-\left(L_{t}+L m_{t}\right) \frac{\partial \mu_{t-1}}{\partial \boldsymbol{\theta}} \\
\frac{\partial \sigma_{t}^{2}}{\partial \mathbf{a}} & =\mathbf{V} \mathbf{a}_{t}+\left(K_{t}+K m_{t}\right) \frac{\partial \sigma_{t-1}^{2}}{\partial \mathbf{a}},
\end{aligned}
$$


for some vectors $V m_{t} \in \mathbb{R}^{5 m+p}, V a_{t} \in \mathbb{R}^{3}$ and scalars $K m_{t}$ and $L m_{t}$.

\section{Proof}

(i) Note that the Gamma distribution $G\left(\sigma_{t-1}^{2} ; r, s\right)$ can be expressed in terms of the so-called (normalized) Incomplete Gamma Integral through the formula

$$
G\left(\sigma_{t-1}^{2} ; r, s\right)=P\left(r, s \sigma_{t-1}^{2}\right)
$$

where $P(a, z)=\frac{1}{\Gamma(a)} \int_{0}^{z} t^{a-1} e^{-t} d t, a, z>0$. The partial derivatives of the Gamma distribution with respect to its parameters are given by

$\frac{\partial G}{\partial r}=g\left(\sigma_{t-1}^{2} ; r, s\right) \frac{\partial \sigma_{t-1}^{2}}{\partial r}+P_{1}\left(r, s \sigma_{t-1}^{2}\right)$ and $\frac{\partial G}{\partial s}=g\left(\sigma_{t-1}^{2} ; r, s\right) \frac{\partial \sigma_{t-1}^{2}}{\partial s}+\frac{1}{s} g\left(\sigma_{t-1}^{2} ; r, s\right) \sigma_{t-1}^{2}$,

where $g(u ; r, s)=\frac{s^{r}}{\Gamma(r)} u^{r-1} e^{-s u}$ and $P_{1}(r, z)=\frac{\partial P(r, z)}{\partial r}$. For computational purposes, the partial derivative of the normalized Incomplete Gamma distribution $P_{1}$ is approximated via numerical procedures as those described in Moore (1982).

By rewriting the conditional variance as

$$
\sigma_{t}^{2}=\alpha_{0}+\left(\beta_{0}+\delta_{0}\left(\frac{u_{t-1}}{\sigma_{t-1}^{2}}-\gamma\right)^{2}\right) \sigma_{t-1}^{2}+\sum_{i=1}^{m-1}\left(\alpha_{i}+\left(\beta_{i}+\delta_{i}\left(\frac{u_{t-1}}{\sigma_{t-1}^{2}}-\gamma\right)^{2}\right) \sigma_{t-1}^{2}\right) G_{i, t}
$$


it is straightforward to verify the recursions $(A .7)$ where

$$
\begin{aligned}
& \mathbf{V}_{t}= {\left[\mathbf{0}_{m+1}^{\prime}, 1, G_{1, t}, \ldots, G_{m-1, t}, \sigma_{t-1}^{2}, \sigma_{t-1}^{2} G_{1, t}, \ldots, \sigma_{t-1}^{2} G_{m-1, t},\right.} \\
&\left(\frac{u_{t-1}}{\sigma_{t-1}^{2}}-\gamma\right)^{2} \sigma_{t-1}^{2},\left(\frac{u_{t-1}}{\sigma_{t-1}^{2}}-\gamma\right)^{2} \sigma_{t-1}^{2} G_{1, t}, \ldots,\left(\frac{u_{t-1}}{\sigma_{t-1}^{2}}-\gamma\right)^{2} \sigma_{t-1}^{2} G_{m-1, t}, \\
& 2\left(\delta_{0}+\sum_{i=1}^{m-1} \delta_{i} G_{i, t}\right)\left(\gamma \sigma_{t-1}^{2}-u_{t-1}\right) \\
&\left(\alpha_{1}+\beta_{1} \sigma_{t-1}^{2}+\delta_{1}\left(\frac{u_{t-1}}{\sigma_{t-1}^{2}}-\gamma\right)^{2} \sigma_{t-1}^{2}\right) D r_{1, t}, \ldots, \\
&\left(\alpha_{m-1}+\beta_{m-1} \sigma_{t-1}^{2}+\delta_{m-1}\left(\frac{u_{t-1}}{\sigma_{t-1}^{2}}-\gamma\right)^{2} \sigma_{t-1}^{2}\right) D r_{m-1, t}, \\
&\left(\alpha_{1}+\beta_{1} \sigma_{t-1}^{2}+\delta_{1}\left(\frac{u_{t-1}}{\sigma_{t-1}^{2}}-\gamma\right)^{2} \sigma_{t-1}^{2}\right) D s_{1, t}, \ldots \\
&\left.\left(\alpha_{m-1}+\beta_{m-1} \sigma_{t-1}^{2}+\delta_{m-1}\left(\frac{u_{t-1}}{\sigma_{t-1}^{2}}-\gamma\right)^{2} \sigma_{t-1}^{2}\right) D s_{m-1, t}\right]^{\prime} \\
& K_{t}=\beta_{0}+\sum_{i=1}^{m-1} \beta_{i} G_{i, t}-\left(\delta_{0}+\sum_{i=1}^{m-1} \delta_{i} G_{i, t}\right)\left(\frac{u_{t-1}}{\sigma_{t-1}^{2}}-\gamma\right)^{2} \\
&+\sum_{i=1}^{m-1}\left(\alpha_{i}+\left(\beta_{i}+\delta_{i}\left(\frac{u_{t-1}}{\sigma_{t-1}^{2}-\gamma}\right)^{2}\right) \sigma_{t-1}^{2}\right) g_{i, t}
\end{aligned}
$$

and

$$
L_{t}=2\left(\delta_{0}+\sum_{i=1}^{m-1} \delta_{i} G_{i, t}\right)\left(\frac{u_{t-1}}{\sigma_{t-1}^{2}}-\gamma\right)
$$

where $\mathbf{0}_{p+1} \in \mathbb{R}^{p+1}, G_{i, t} \equiv G\left(\sigma_{t-1}^{2} ; r_{i}, s_{i}\right), g_{i, t} \equiv g\left(\sigma_{t-1}^{2} ; r_{i}, s_{i}\right), D r_{i, t} \equiv P_{1}\left(r_{i}, s_{i} \sigma_{t-1}^{2}\right)$ and $D s_{i, t} \equiv \frac{1}{s_{i}} g_{i, t} \sigma_{t-1}^{2}$, for $i=1, \ldots, m-1$. Finally, the second recursive formula in (A.7) is verified for

$$
\mathbf{E}_{t}=\left[1, x_{t-1}, \ldots, x_{t-p}, \mathbf{0}_{5 m-1}^{\prime}\right]^{\prime},
$$

with $\mathbf{0}_{5 m-1} \in \mathbb{R}^{5 m-1}$.

(ii) The gradient for the extra regime expansion (21) can be directly obtained as in part (i) by noting that for any component $\theta_{j}$ in $\boldsymbol{\theta}$, the corresponding partial derivative takes the form

$$
\frac{\partial \sigma_{t}^{2}}{\partial \theta_{j}}=V_{j, t}+V m_{j, t}+\left(K_{t}+K m_{t}\right) \frac{\partial \sigma_{t-1}^{2}}{\partial \theta_{j}}+\left(L_{t}+L m_{t}\right) \frac{\partial u_{t-1}}{\partial \theta_{j}}
$$


where

$$
\begin{gathered}
V m_{j, t}=2 a_{3}\left(\gamma \sigma_{t-1}^{2}-u_{t-1}\right) \sigma_{t-1}^{4} 1_{\left\{\theta_{j}=\gamma\right\}} \\
K m_{t}=\left[2 a_{1} / \sigma_{t-1}^{2}+3 a_{2}-a_{3}\left(\frac{u_{t-1}}{\sigma_{t-1}^{2}}-\gamma\right)\left(\frac{u_{t-1}}{\sigma_{t-1}^{2}}-3 \gamma\right)\right] \sigma_{t-1}^{4},
\end{gathered}
$$

and

$$
L m_{t}=2 a_{3}\left(\frac{u_{t-1}}{\sigma_{t-1}^{2}}-\gamma\right) \sigma_{t-1}^{4}
$$

Equation $(A .8)$ is then obtained by considering the vectors $\mathbf{V}_{t}=\left(V_{j, t}\right)$ and $\mathbf{V m}_{t}=\left(V m_{j, t}\right)$. Meanwhile, to verify equation $(A .9)$, it can be shown that the partial derivative with respect to $a_{i}$ is given by

$$
\frac{\partial \sigma_{t}^{2}}{\partial a_{i}}=V a_{i, t}+\left(K_{t}+K m_{t}\right) \frac{\partial \sigma_{t-1}^{2}}{\partial a_{i}}
$$

where $V a_{i, t}$ is the $i$ th element in vector

$$
\mathbf{V} \mathbf{a}_{t}=\left(\sigma_{t-1}^{4}, \sigma_{t-1}^{6},\left(u_{t-1}-\gamma \sigma_{t-1}^{2}\right)^{2} \sigma_{t-1}^{2}\right)^{\prime}
$$

Lemma 3. Let $r>0$ and consider $\gamma(r, z)=\int_{0}^{z} u^{r-1} e^{-u} d u, z \geq 0$ the so-called lower incomplete gamma function. Given $r$ and $M>0$, there are positive number $s^{*}=s^{*}(r, M)$ and $k=k(r, s, M)$ such that for all $0<x<M$

$$
\gamma(r, s x)=c_{r} s^{k} x^{2}+R, 0<s<s^{*}
$$

where $|R|<s^{k} M^{2}$ and $c_{r}=\frac{1}{2 r}$ if $r \leq 1$ and $c_{r}=\frac{1}{2}$ otherwise.

\section{Proof}

Let $r, M>0$ and define the function $f(x)=x^{r-1} e^{-x}, x \geq 0$.

We first suppose $r>1$. Since $s^{r} e^{-s M}>0$ is bounded as function of $s$ and strictly 
increasing in the interval $(0, r / M]$, it is implied that there is $s_{1}=s_{1}(r, M)$ such that

$$
s^{r} e^{-s M}<(1 / M)^{r-2}, 0<s<s_{1} .
$$

Define

$$
k(r, s, M)=r+((r-2) \log M-s M) / \log s, 0<s<s_{2},
$$

where $s_{2}=\min \left(s_{1}, 1,\right)$.It is important to remark that the values of $k$ given by $(A .20)$ are always positive. From equations (A.19) and (A.20), it is implied that

$$
(s M)^{r-1} e^{-s M}=s^{k-2}(s M),
$$

i.e. the straight line $g(x)=s^{k-2} x$ intersects $f(x)$ at the point $s M$.

When $r>2$, it is straightforward to verify that $f(x)$ is convex in the interval $(0, s M)$ for all $s<s^{*}=\min \left(s_{2}, \frac{r-1-\sqrt{r-1}}{M}\right)$. For $0<\frac{t}{M}<1$, we have that $f((t / M) s M+(1-t / M) 0)<$ $(t / M) g(s M)$, i.e.

$$
f(s t)<s^{k-1} t, 0<t<M
$$

By integrating both sides in equality A.21 with respect to $t$ from 0 to $x<M$, we obtain that

$$
\int_{0}^{s x} f(u) d u<\frac{s^{k}}{2} x^{2}, x<M
$$

Meanwhile, when $1<r \leq 2,-f(x)$ is convex so that for all $s<s^{*}=s_{2}$

$$
\frac{s^{k}}{2} x^{2}<\int_{0}^{s x} f(u) d u, x<M
$$

Both left and right sides in inequalities (A.22) and (A.23) are bounded by $\frac{1}{2} s^{k} M^{2}$ and $s^{k} M^{2}$, respectively. Therefore, the error of approximating $\int_{0}^{s x} f(u) d u$ by $\frac{s^{k}}{2} x^{2}$ is bounded by the 
last quantity

$$
|R|=\left|\int_{0}^{s x} f(u) d u-\frac{s^{k}}{2} x^{2}\right|<s^{k} M^{2} .
$$

For the remaining case $0<r \leq 1$, a similar bound can be derived by noting that through integrating by parts $\gamma(r, z)=\frac{1}{r}\left(s^{r} x^{r} e^{-s x}+\gamma(r+1, z)\right)$, so that $(A .24)$ is bounded by $\frac{1}{r}\left(s^{r} M^{r}+s^{k^{*}} M^{2}\right)=s^{k} M^{2}$ for some $k>k^{*}$, where $k^{*}$ is given by $(A .20)$ with $r+1$ instead of $r$. 


\section{References}

[1] Andersen, T. G. and T. Bollerslev. 1998. Answering the Skeptics:Yes, Standard volatility Models do Provide Accurate Forecasts. International Economic Review 39: 885-905.

[2] Berndt, E.R., B.H. Hall, R.E. Hall and J.A. Hausman. 1974. Estimation and Inference in Nonlinear Structural Models. Annals of Economic and Social Measurement 3: 653-665.

[3] Black, F. 1976. Studies of Stock Price Volatility Changes. In Proceedings of the 1976 Meetings of the Business and Economics Statistics Section, American Statistical Association: 177-181.

[4] Bollerslev, T. 1986. Generalized Autoregressive Conditional Heteroskedasticity. Journal of Econometrics 21: 307-328.

[5] Christie, A. 1982. The Stochastic Behavior of Common Stock Variances: Value, Leverage and Interest Rate Effects. Journal of Financial Economics 10: 407-432.

[6] Christoffersen, P.F. 1998. Evaluating Interval Forecasts. International Economic Review 39: 841-862.

[7] Cox, J., J. Ingersoll, and S. Ross. 1985. A Theory of the Term Structure of Interest Rates. Econometrica 53: 385-407.

[8] Ding, Z., C.W.J. Granger and R.F. Engle. 1993. A Long Memory Property of Stock Market Returns and a New Model. Journal of Empirical Finance 1: 83-106.

[9] Engle, R.F. 1982.Autoregressive Conditional Heteroskedasticity with Estimates of the Variance of the UK Inflation. Econometrica 50: 987-1007.

[10] Engle, R. F. and C. Mustafa. 1992. Implied ARCH Models from Options Prices. Journal of Econometrics 52: 289-311. 
[11] Engle, R. and V. Ng. 1993. Measuring and Testing the Impact of News on Volatility. Journal of Finance 43: 1749-1778.

[12] Feller, W. 1951. Two Singular Diffusion Problems. Annals of Mathematics 54: 173-182.

[13] Fornari, F. and A. Mele. 1997. Sign- and Volatility-Switching ARCH Models: Theory and Applications to International Stock Markets. Journal of Applied Econometrics 12: 49-65.

[14] Glosten, L., R. Jagannanthan and R. Runkle. 1993. On the Relationship Between the Expected Value and the Volatility of the Nominal Excess Returns on Stocks. Journal of Finance 48: 1779-1801.

[15] Goldfeld, S.M. and R.E. Quandt. 1973. A Markov Model for Switching Regression. Journal of Econometrics 1: 3-16.

[16] Hamilton, J.D. 1989. A New Approach to the Economic Analysis of Nonstationary Time Series and the Business Cycle. Econometrica 57: 357-384.

[17] Hansen, P.R. 2005. A Test for Superior Predictive Ability. Journal of Business $E$ Economic Statistics 23: 365-380.

[18] Hansen, P.R. and A. Lunde. 2005. A Forecast Comparison of Volatility Models: Does Anything Beat a GARCH $(1,1)$ ?. Journal of Applied Econometrics 20: 873-889.

[19] Heston, S. and S. Nandi. 2000. A Closed-Form GARCH Option Valuation Model. The Review of Financial Studies 13: 585-625.

[20] Lundbergh, S. and T. Teräsvirta. 2002. Evaluating GARCH Models. Journal of Econometrics 110: 417-435.

[21] Luukkonen, R., P. Saikkonen and T. Teräsvirta. 1988. Testing Linearity against Smooth Transition Autorregresive Models. Biometrika 75: 491-499. 
[22] Mebane W.R. and J.S. Sekhon. 2011. Genetic Optimization using Derivatives: The rgenoud package for R. Journal of Statistical Software 42(11): 1-26. URL http://www.jstatsoft.org/v42/i11

[23] Medeiros, M.C. and A. Veiga. 2009. Modeling Multiple Regimes in Financial Volatility with a Flexible Coefficient GARCH(1,1) Model. Econometric Theory 25: 117-161.

[24] Meitz, M. and P. Saikkonen. 2008a. Stability of Nonlinear AR-GARCH Models. Journal of Time Series Analysis 29: 453-475.

[25] Meitz, M. and P. Saikkonen. 2008b. Ergodicity Mixing, and Existence of Moments of a Class of Markov Models with Applications to GARCH and ACD models. Econometric Theory 24: 1291-1320.

[26] Meitz, M. and P. Saikkonen. 2010. "Parameter Estimation in Nonlinear AR-GARCH Models". Tüsiad-Koc University Economic Research Forum Working Papers 1002, Tüsiad-Koc University Economic Research Forum.

[27] Moore, R.J. 1982. Algorithm AS 187: Derivatives of the Incomplete Gamma Integral. Journal of the Royal Statistical Society: Series C (Applied Statistics) 31: 330-335.

[28] Nelson, D.B. 1991. Conditional Heteroskedasticity in Asset Returns: A new approach. Econometrica 59: 347-370.

[29] Silvennoinen, A. and T. Terasvirta. 2009. Modeling Multivariate Autoregressive Conditional Heteroskedasticity with the Double Smooth Transition Conditional Correlation GARCH model. Journal of Financial Econometrics 7: 373-411.

[30] van Dijk, D., P.H. Franses and A. Lucas. 1999. Testing for Smooth Transition Nonlinearity in the Presence of Outliers. Journal of Applied Econometrics 14: 539-562.

[31] Wooldridge, J.M. 1990.A Unified Approach to Robust, Regression-Based Specification Tests. Econometric Theory 6: 17-43. 


\section{$7 \quad$ Notes}

1. Given the parameters $s_{1}>s_{2}$ and $r_{1} \leq r_{2}$, Assumption $3(i i)$ is a consequence of the dominance relationship between the Gamma densities $g\left(u ; r_{1}, s_{1}\right) \geq g\left(u ; r_{2}, s_{2}\right)$ which is equivalent to $e^{-\left(s_{1}-s_{2}\right) u} \geq c u^{r_{2}-r_{1}}$, where $c \equiv \frac{\Gamma\left(r_{1}\right) s_{2}^{r_{2}}}{\Gamma\left(r_{2}\right) s_{1}^{r_{1}}}$. The inequality holds for any $u$ in the non-empty interval $\left(0, x^{*}\right]$ where $x^{*}$ is the solution of the equation $h(x) \equiv e^{-\left(s_{1}-s_{2}\right) x}-$ $c x^{r_{2}-r_{1}}=0$, which exits because $h$ is continuous and $h\left(c^{*}\right) \leq 0<h(0)$, where $c^{*} \equiv$ $\max \left\{1, e^{-\left(s_{1}-s_{2}\right) /\left(r_{2}-r_{1}\right)} c^{-1 /\left(r_{2}-r_{1}\right)}\right\}$.

2. Letting $t=s u$ in (14), the Gamma distribution is rewritten as $G(x ; r, s)=\frac{1}{\Gamma(r)} \int_{0}^{s x} t^{r} e^{-t} d t=$ $\frac{1}{\Gamma(r)} \gamma(r, s x)$, where $\gamma(r, z)$ is the so-called lower incomplete gamma function. The absolute value of the remainder is bounded by $\frac{c_{r}}{\Gamma(r)} s^{k} M^{2}$ and tends to zero as $s \rightarrow 0$.

3. http://online.thomsonreuters.com/datastream/ 


\begin{tabular}{|c|c|c|c|c|c|c|c|c|c|c|}
\hline \multirow[b]{2}{*}{ Market } & \multicolumn{5}{|c|}{ Stock Index } & \multicolumn{5}{|c|}{ FX rate to USD } \\
\hline & Series & mean & std & $s k$ & $k u$ & Series & mean & std & $s k$ & $k u$ \\
\hline Netherlands & AEX & 0.0002 & 0.0145 & -0.1189 & 6.9 & EUR & 0.0000 & 0.0060 & -0.0762 & 3.9 \\
\hline Austria & ATX & 0.0004 & 0.0111 & -0.7861 & 7.9 & $"$ & $"$ & $"$ & $"$ & $"$ \\
\hline France & CAC40 & 0.0003 & 0.0139 & -0.1420 & 5.8 & $"$ & $"$ & $"$ & $"$ & $"$ \\
\hline Germany & DAX30 & 0.0003 & 0.0153 & -0.2620 & 6.1 & $"$ & $"$ & $"$ & $"$ & $"$ \\
\hline Spain & IBEX35 & 0.0003 & 0.0136 & -0.2160 & 6.0 & $"$ & $"$ & $"$ & $"$ & $"$ \\
\hline South Africa & ALSI & 0.0005 & 0.0122 & -0.8890 & 12.4 & ZAR & 0.0002 & 0.0102 & -0.0261 & 16.4 \\
\hline Australia & ASX200 & 0.0003 & 0.0087 & -0.4962 & 9.1 & AUD & 0.0000 & 0.0070 & 0.0579 & 5.7 \\
\hline India & BSE100 & 0.0005 & 0.0168 & -0.4193 & 6.7 & INR & 0.0001 & 0.0029 & 1.3732 & 51.5 \\
\hline UK & FTSE100 & 0.0001 & 0.0113 & -0.1867 & 5.6 & GBP & 0.0000 & 0.0051 & 0.0023 & 5.7 \\
\hline Brazil & IBOVESPA & 0.0007 & 0.0218 & 0.3745 & 18.1 & BRL & 0.0002 & 0.0097 & 0.5594 & 27.4 \\
\hline Mexico & IPC & 0.0007 & 0.0153 & -0.0563 & 10.2 & MXN & 0.0001 & 0.0058 & 0.9534 & 16.6 \\
\hline Turkey & ISE100 & 0.0013 & 0.0278 & -0.0444 & 8.1 & TRY & 0.0008 & 0.0115 & 4.5190 & 90.3 \\
\hline Japan & NIKKEI225 & -0.0001 & 0.0142 & -0.0963 & 5.0 & JPY & 0.0000 & 0.0068 & -0.7664 & 10.8 \\
\hline Russia & RTSI & 0.0007 & 0.0258 & -0.5290 & 10.1 & RUB & 0.0005 & 0.0189 & 5.5241 & 284.4 \\
\hline Thailand & SET & -0.0001 & 0.0172 & 0.2783 & 9.9 & THB & 0.0001 & 0.0069 & 0.7929 & 40.3 \\
\hline Switzerland & SMI & 0.0002 & 0.0122 & -0.1870 & 7.0 & CHF & 0.0000 & 0.0066 & -0.1466 & 4.0 \\
\hline Canada & TSX & 0.0003 & 0.0100 & -0.6646 & 8.1 & CAD & -0.0001 & 0.0045 & 0.0626 & 5.1 \\
\hline USA & S\&P500 & 0.0002 & 0.0112 & -0.0874 & 6.0 & & & & & \\
\hline
\end{tabular}

Table 1. Descriptive statistics of daily returns 


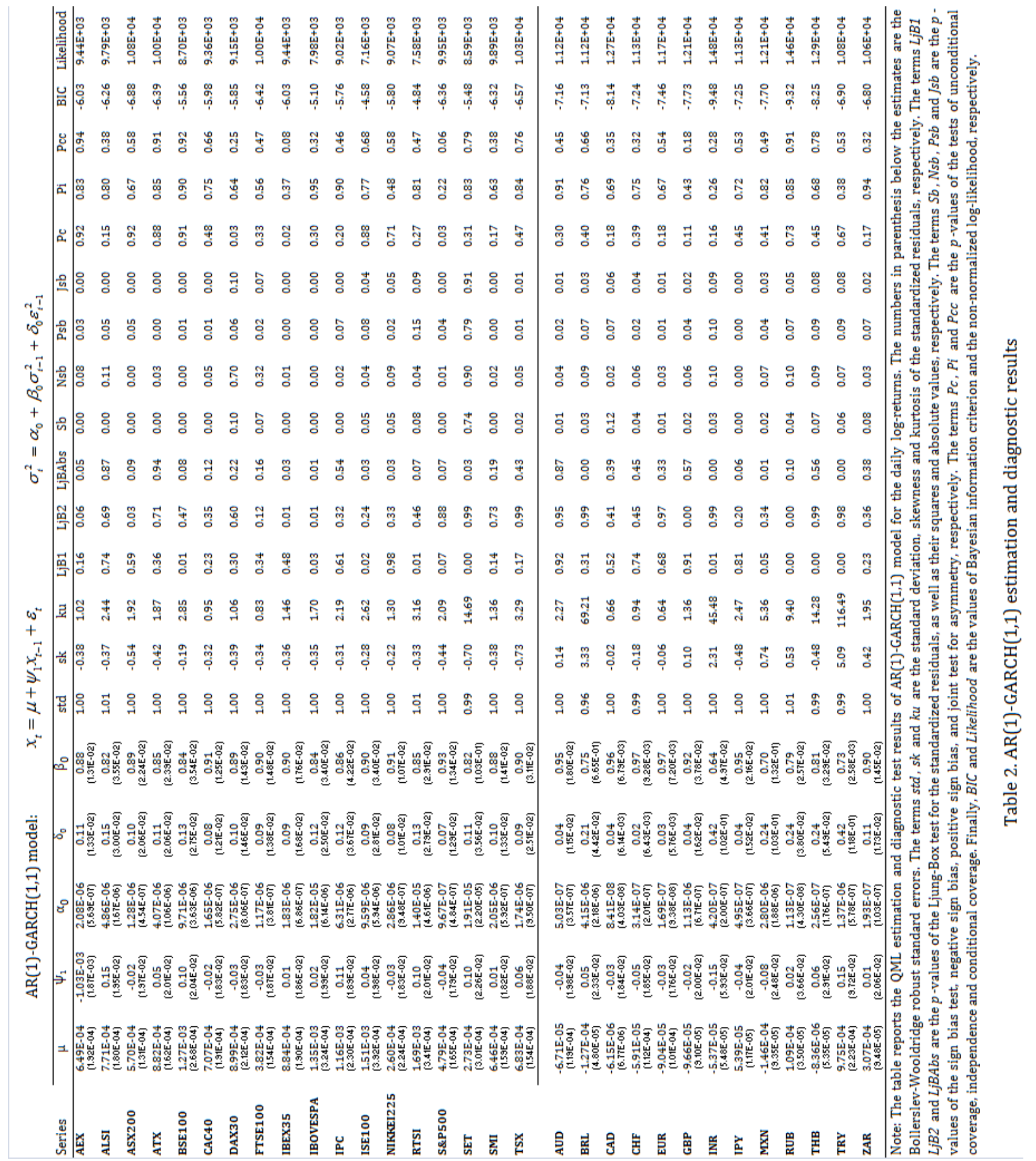




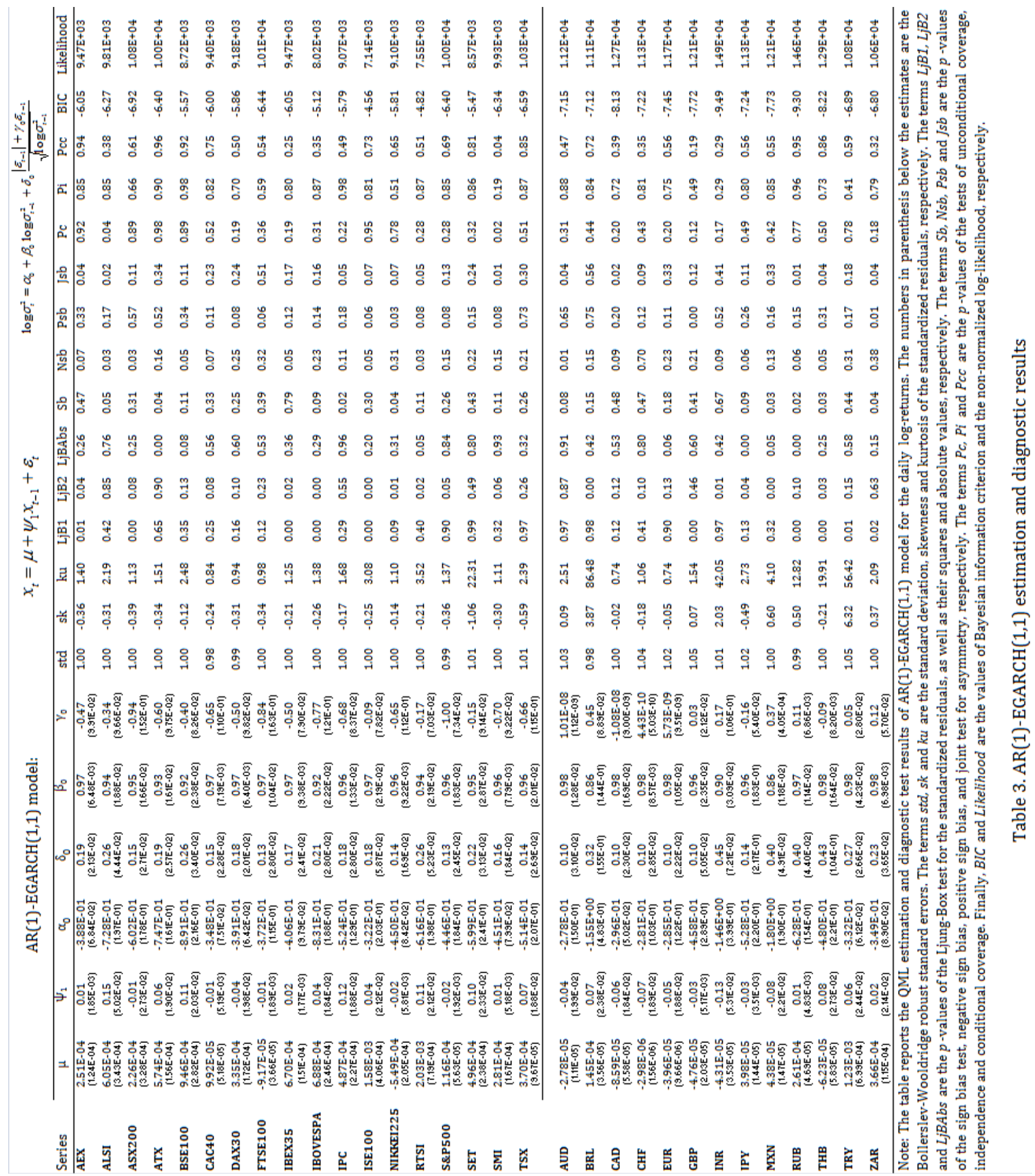




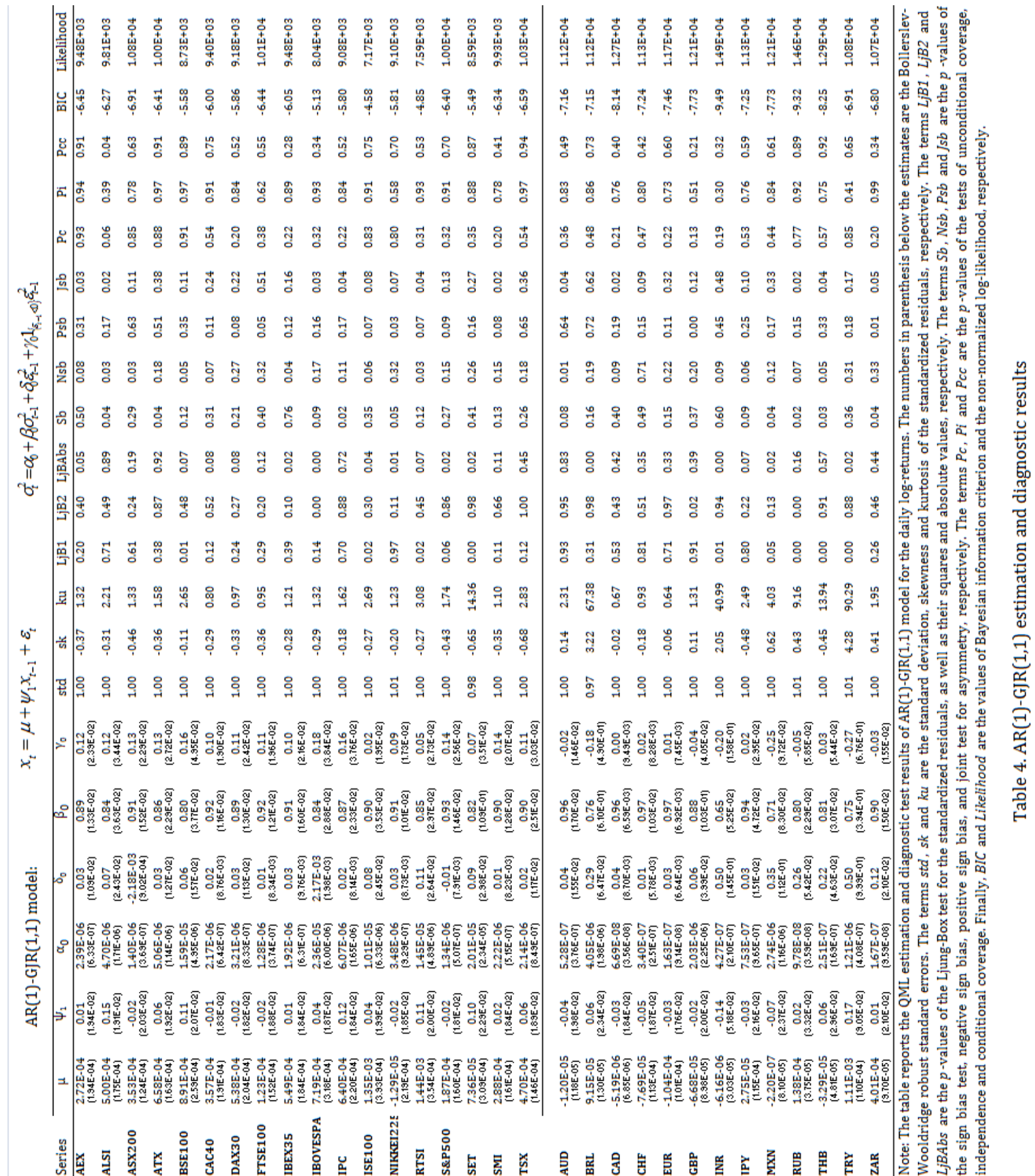




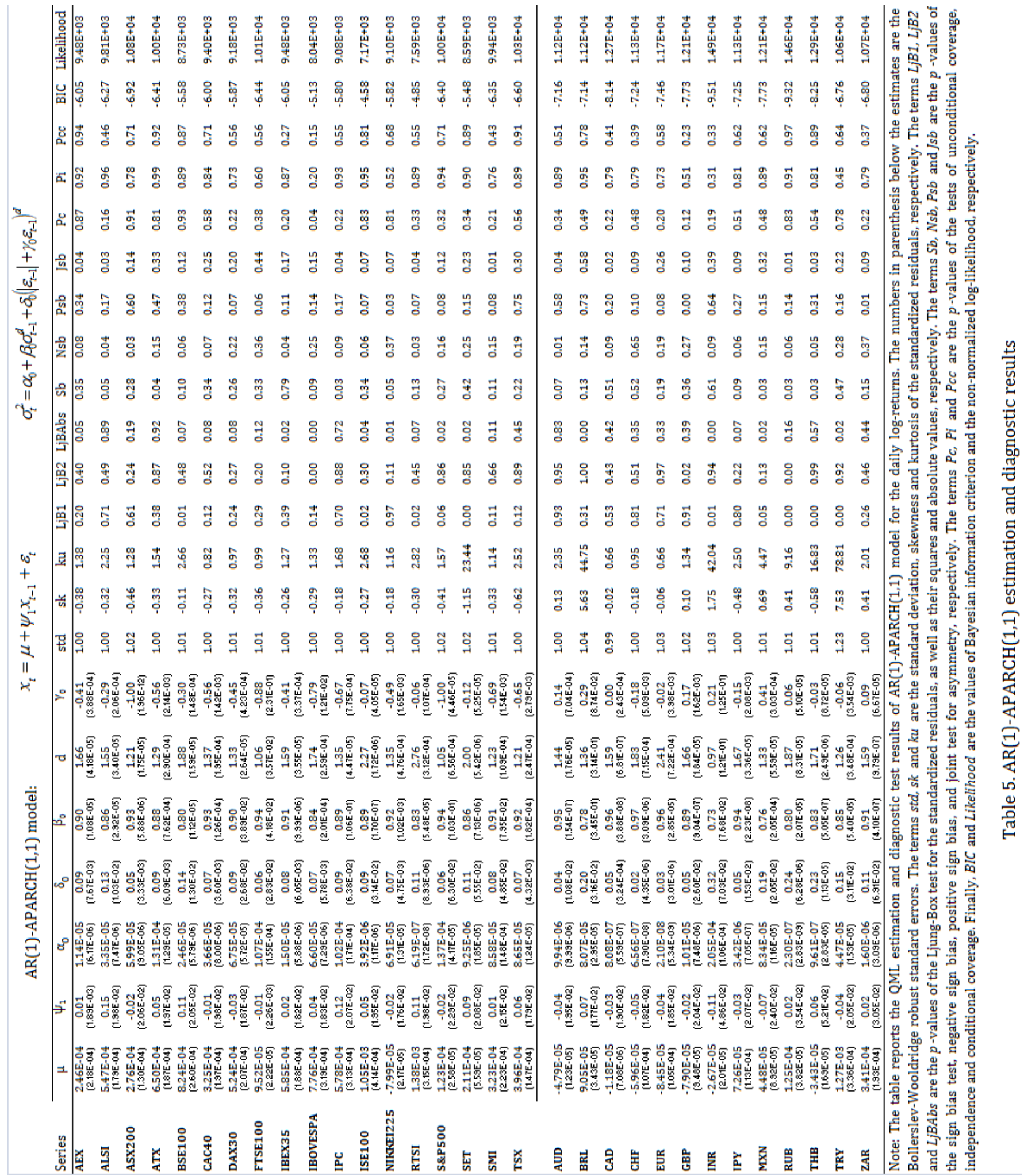




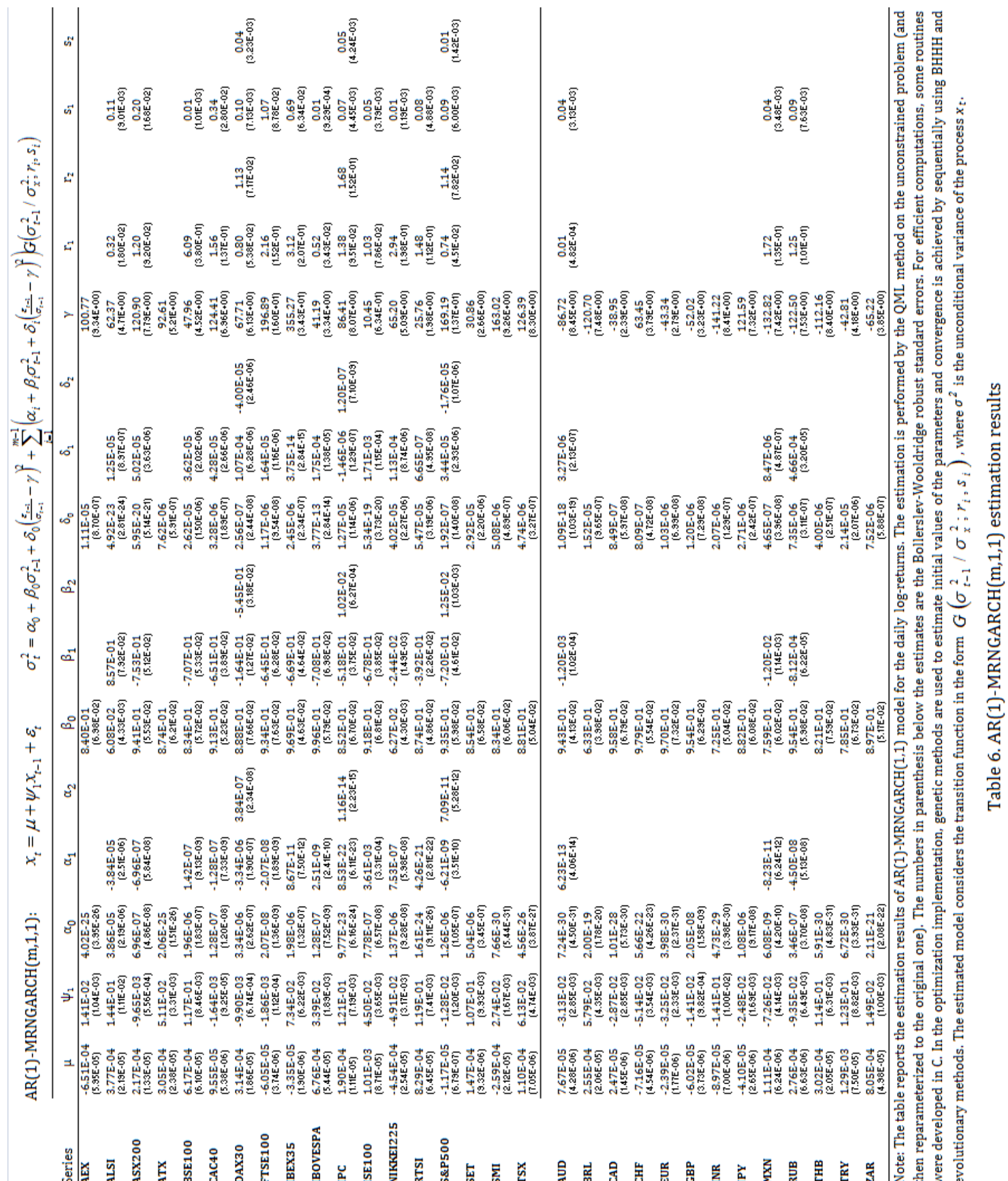




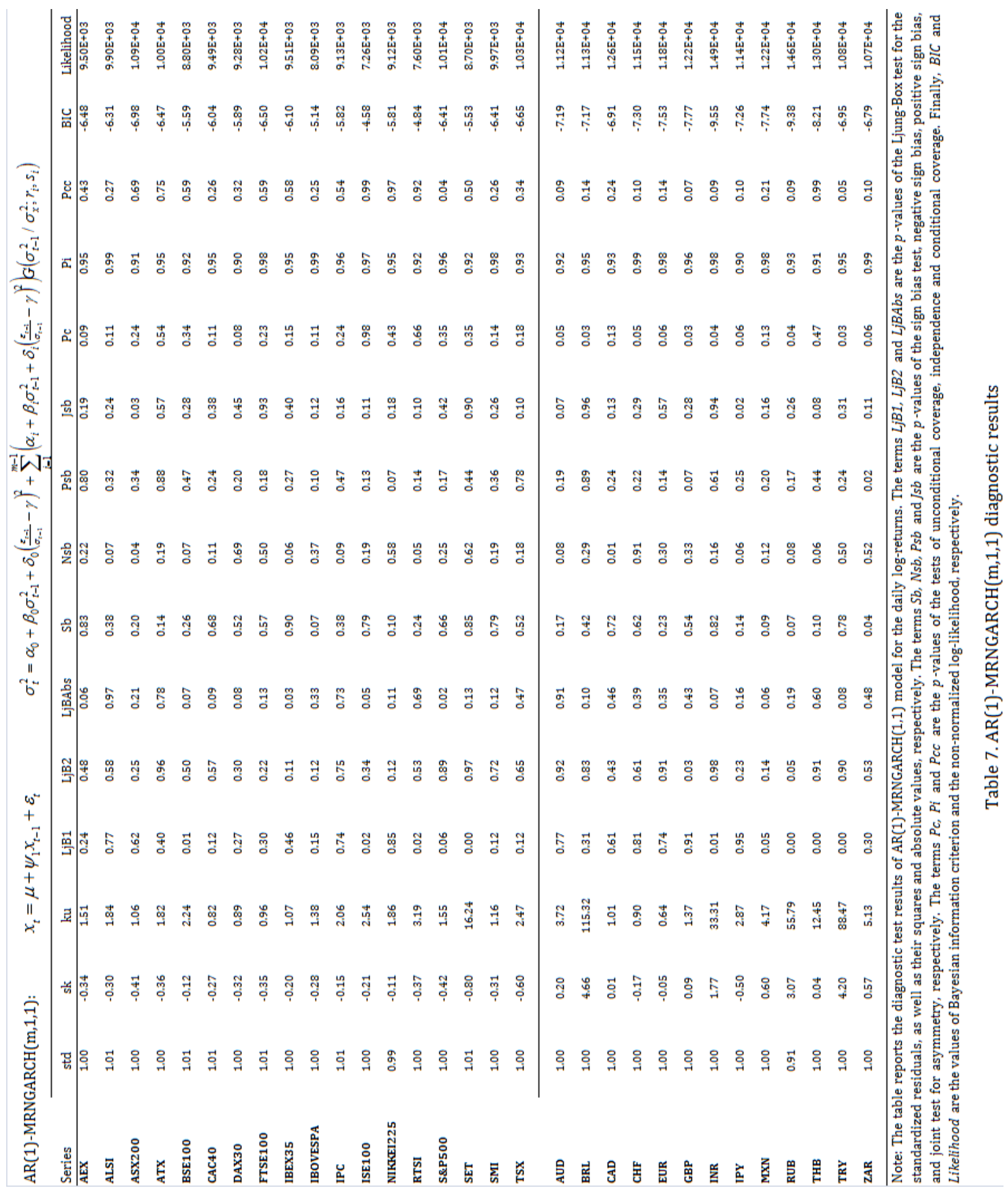




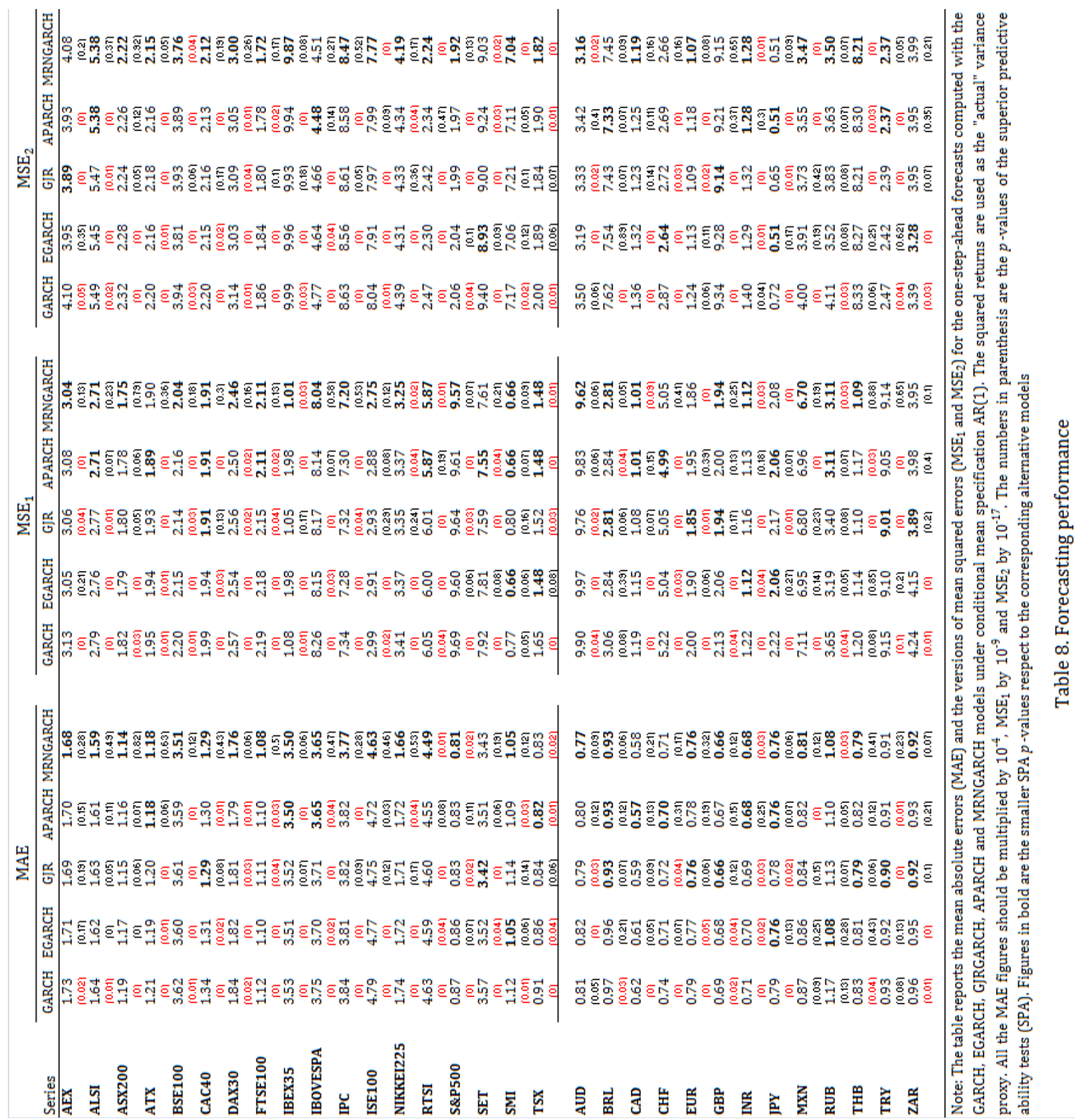

